\title{
Mass segregation in very young open clusters - A case study of NGC 2244 and NGC 6530
}

\author{
L. Chen \\ Shanghai Astronomical Observatory, Chinese Academy of Sciences, 80 Nandan Road, \\ Shanghai 200030, P.R. China \\ chenli@shao.ac.cn \\ R. de Grijs \\ Department of Physics and Astronomy, The University of Sheffield, Hicks Building, \\ Hounsfield Road, Sheffield S3 7RH, U.K.; and National Astronomical Observatories, \\ Chinese Academy of Sciences, 20A Datun Road, Chaoyang District, Beijing 100012, P.R. \\ China \\ R.deGrijs@sheffield.ac.uk \\ and \\ J. L. Zhao \\ Shanghai Astronomical Observatory, Chinese Academy of Sciences, 80 Nandan Road, \\ Shanghai 200030, P.R. China
}

\begin{abstract}
We derive the proper motions, membership probabilities, and velocity dispersions of stars in the regions of the young ( $\sim 2-4$ Myr-old) open clusters NGC 2244 (the central cluster in the Monoceros R2 association) and NGC 6530 (the dominant cluster in the Sgr OB1 association) from photographic plate material obtained at Shanghai Astronomical Observatory, with time baselines of 34 and 87 years, respectively. Both clusters show clear evidence of mass segregation, but they do not exhibit any significant velocity-mass (or, equivalently, a velocityluminosity) dependence. This provides strong support for the suggestion that the observed mass segregation is - at least partially - due to the way in which star formation has proceeded in these complex star-forming regions ("primordial" mass segregation). Based on arguments related to the clusters' published initial mass functions, in conjunction with our new measurements of their internal velocity dispersions ( $\sim 35$ and $8 \mathrm{~km} \mathrm{~s}^{-1}$ for NGC 2244 and NGC 6530,
\end{abstract}


respectively), we provide strong arguments in favor of the dissolution of NGC 2244 on very short time-scales, while we speculate that NGC 6530 may be more stable against the effects of internal two-body relaxation. However, this latter object may well be destroyed by the strong tidal field prevalent at its location in the Galactic plane in the direction of the Galactic Center.

Subject headings: astrometry — stellar dynamics — open clusters: general open clusters: individual (NGC 2244, NGC 6530)

\section{Introduction}

Open clusters are considered excellent laboratories for studies of stellar evolution and the dynamics of stellar systems. A large fraction of Galactic open clusters are situated close to the Galactic plane, thus potentially making them appropriate objects for probing the structure and evolution of the Galactic disc - although the presence of dust in the Galactic plane restricts their usefulness in this sense.

One of many important aspects in open cluster studies is related to the effects of mass segregation; that is, the more massive member stars in the cluster will be more centrally concentrated and/or have a distinct distribution in velocity space compared to the lowermass members. The key observational questions of interest in open star cluster research include whether or not mass segregation exists in a given star cluster and, if so, what the extent of this effect is in relation to the cluster's age. For cluster samples as a whole, the similarities and differences of the effects of mass segregation for clusters of various ages, abundances and galactocentric distances are of great interest. All of these properties are related to the formation process as well as the dynamical evolution of clusters. They are also (sometimes significantly) influenced by the Galactic tidal fields, in particular in the outer regions of a given cluster.

Young open clusters are important stellar systems for our understanding of the starformation process. In very young clusters, most of the low-mass stars will still be in their pre-main-sequence (PMS) evolutionary stage, and one may expect to derive global stellar initial mass functions (IMFs) that are not yet significantly affected by the effects of stellar and dynamical evolution. However, if a significant amount of "primordial" mass segregation (i.e., mass segregation intrinsic to the star-formation process itself) is present, this would seriously complicate the interpretation of an observed stellar luminosity function (LF) at a given position within a star cluster in terms of its IMF (de Grijs et al. 2002a, b).

In this paper, we use photographic plate material with longer time baselines than pub- 
lished previously, in order to determine the proper motions and membership probabilities of stars in the regions of two very young Galactic open clusters NGC 2244 and NGC 6530. We aim to quantify the possible effects of mass segregation and place these objects in the context of their expected future evolution.

\subsection{NGC 2244}

NGC 2244 (also referred to as NGC 2239) is the core OB cluster of the Monoceros OB2 (Mon OB2) association, embedded in the Rosette Nebula (NGC 2237/2246), a welldeveloped "blister" His region. This system is located in the northwest quadrant of the Rosette Molecular Cloud complex, which itself is one of the most massive giant molecular cloud (GMC) complexes in the Milky Way (see Li \& Smith (2005b)); such GMC complexes are particularly conducive to the formation of massive OB stars. Because of the strong ionizing radiation from the massive OB stars in the core of NGC 2244 (see, e.g., Pérez (1987) for a census of the OB stars in the cluster), the cluster has excavated the GMC complex, allowing us a deeper look into the cloud than otherwise possible. NGC 2244 represents the youngest of two or three subgroups of OB stars and stellar aggregates in the Mon OB2 association (Li 2005; Li \& Smith 2005b).

Since NGC 2244 is one of the youngest star-forming open clusters in the Milky Way, and relatively nearby, it has been the subject of a number of detailed studies. Using the WEBDA open cluster database (Mermilliod \& Paunzen 2003) as our guide, the equatorial coordinates of the cluster center are $(\alpha, \delta)_{2000}=\left(06^{\mathrm{h}} 31^{\mathrm{m}} 55^{\mathrm{s}},+04^{\circ} 56^{\prime} 30^{\prime \prime}\right)$, and the Galactic coordinates $(l, b)=\left(206^{\circ} .31,-2^{\circ} .07\right)$, i.e., the cluster is located quite close to the direction of the Galactic anticenter.

Li (2005), using near-infrared imaging from the Two Micron All-Sky Survey (2MASS), estimated a mean visual extinction, $A_{V} \simeq 1.5 \mathrm{mag}$ within a radius of 10 arcmin centered on the apparent center of the His region. They pointed out that this independent low nearinfrared extinction estimate was consistent with previous estimates in the optical, which is also supported by results from CO and $\mathrm{C}^{13} \mathrm{O}$ maps (e.g., Blitz \& Stark (1986); Williams et al. (1995)). In the optical, the most comprehensive extinction analysis for the cluster was published by Massey et al. (1995), who reported a mean reddening of $E(B-V)=0.48 \mathrm{mag}$, with a non-negligible amount of differential reddening across the cluster: their reddening values for individual cluster stars range from $E(B-V)=0.08$ to 0.98 mag. This is fully consistent with the results of Park \& Sung (2002); and references therein), who found $\langle E(B-$ $V)\rangle=0.47 \pm 0.04 \mathrm{mag}$ from the individual reddening values for 28 cluster member stars brighter than $V=14 \mathrm{mag}$, and a smaller amount of differential reddening, ranging from 
$E(B-V)=0.40$ to 0.56 mag. They also found that NGC 2244 is most likely characterized by a "standard" reddening law, with a total-to-selective extinction ratio of $R_{V}=3.1 \pm 0.2$. As such, we adopt a foregound reddening of $E(B-V)=0.48 \mathrm{mag}$ in this paper.

The distance to NGC 2244 is reasonably well established, at $1.4 \lesssim D \lesssim 1.7 \mathrm{kpc}$, and generally consistent among different distance indicators used in the literature (see also Park \& Sung (2002) for a review). For instance, Pérez (1987) obtained $D=1670 \pm 125$ pc from the distance moduli of the individual OB-type stars in the cluster. More recently, Hensberge et al. (2000) derived a distance of $D=1.39 \pm 0.1 \mathrm{kpc}$ to the cluster based on spectroscopic observations of one of the cluster member stars, the eclipsing binary V578 Mon (HD259135). Park \& Sung (2002) derived a distance modulus of $(m-M)_{V, 0}=11.1$ based on $U B V I$ photometry, corresponding to $D=1.7 \mathrm{kpc}$. This larger distance value seems to be favoured by many authors in the field (e.g., Ogura \& Ishida (1981); Sabogal-Martínez et al. (2001)), and as such we will adopt it here as well.

For the age of NGC 2244, most authors agree on a value of 2-4 Myr for the main sequence (MS) turn-off age (Turner (1976); Ogura \& Ishida (1981); Marschall et al. (1982); Pérez et al. (1989); Pérez (1991); Massey et al. (1995); Park \& Sung (2002) and references therein). In addition, recently an age estimate of $2.3 \pm 0.2 \mathrm{Myr}$ was derived from the age of the eclipsing binary V578 Mon (Hensberge et al. 2000). Based on high spatial resolution multi-filter photometry of NGC 2244, Berghöfer \& Christian (2002) concluded that while most of the cluster stars are aged up to $3 \mathrm{Myr}$, significantly younger low-mass stars $(5 \%$ or more of the cluster stars) exist in the cluster as well, indicating that star formation is still in progress in the Rosette Nebula/NGC 2244 region (see also Pérez (1987); Pérez et al. (1989); Park \& Sung (2002), the latter authors found a MS turn-off age of 1.9 Myr, but a pre-MS age spread of about $6 \mathrm{Myr}$ ). However, they also note that up to $45 \%$ of the cluster stars may be older than $3 \mathrm{Myr}$, thus suggesting an earlier star-forming episode in the region. Moreover, the dynamical age of the nebula is just 0.2-0.6 Myr (Matthews 1967), while the dynamical age of the Hi shell is as old as 4 Myr (see Li \& Smith (2005a)).

The first proper motion study of this cluster (van Schewick 1958) identified 22 probable members. Subsequently, Marschall et al. (1982) performed more precise proper motion measurements of 287 stars down to $V=14$ mag and selected 89 stars with membership probabilities, $p \geq 0.7$, and data quality, $q \geq 0.9$, in an area of $35 \times 44 \operatorname{arcmin}^{2}$. However, Park \& Sung (2002) cautioned that their "member" sample may contain several non-cluster members of the Mon OB2 association because of the large rectangular field of view employed by the Marschall et al. (1982) study compared to the cluster's angular size of $\sim 24$ arcmin and the roughly spherical shape described by Pérez (1991). Using the Marschall et al. (1982) astrometric membership determinations, combined with spectroscopically determined stellar 
MK classifications of 193 stars by Verschueren (1991), Park \& Sung (2002) redetermined the membership probabilities of the brightest cluster stars (30 OB stars and 21 pre-MS members and candidates brighter than $V=18 \mathrm{mag}$ ).

Sabogal-Martínez et al. (2001) and Higuera et al. (2002) redetermined the cluster's stellar membership probabilities based on the early astrometric data first analyzed by Marschall et al. (1982). They confirmed 92 (out of 202) stars as physical members of the cluster, with $p>0.5$. In this paper, we will analyze high-quality, wide-field photographic plates of Shanghai Astronomical Observatory, with an maximum likelihood algorithm involving careful assessment of the distributions of both the proper motions and the positions, thus allowing us to arrive at a valuable, independent estimation of membership probabilities.

\subsection{NGC 6530}

NGC 6530 is another prime example of an extremely young open cluster that has been the subject of a number of previous studies. As the core cluster of the Sgr OB1 association, it is projected onto the eastern part of the Lagoon Nebula (M8), which is one of the brightest nearby nebulae and Hir regions (Rauw et al. 2002). The Lagoon Nebula is the illuminated part of the GMC from which the cluster formed by generating a "blister" Hil region due to the ionizing radiation from its hot massive stars (e.g., Lada et al. (1976). Once again, this allows us to probe into the depths of this GMC region much better than would have been possible otherwise.

The equatorial coordinates of NGC 6530 are $(\alpha, \delta)_{2000}=\left(18^{\mathrm{h}} 04^{\mathrm{m}} 31^{\mathrm{s}},-24^{\circ} 21^{\prime} 30^{\prime \prime}\right)$ and the Galactic coordinates $(l, b)=\left(6^{\circ} .083,-1^{\circ} .331\right)$, i.e., the cluster is located in the direction not far from that of the Galactic Center. This implies that one has to be very careful in disentangling cluster members from field interlopers (see also Sung et al. (2000). As a case in point, in early work on this cluster, Walker (1957) obtained photo-electric magnitudes and colors for 118 stars in the NGC 6530 region and proposed that a band of its low-mass members above the MS in color-magnitude space may be PMS stars that are still in the stage of gravitational contraction. It has since been shown (e.g., The (1960); van Altena \& Jones (1972); Chini \& Neckel (1981)) that many of these PMS candidates are, in fact, foregound or background field stars (see the discussions in van den Ancker et al. (1997), and Sung et al. $(2000))$.

Subsequently, the properties of this very young cluster have been studied extensively by different authors, leading to an age estimate of 1.5-3.0 Myr (van Altena \& Jones 1972; Sagar 1978; Sung et al. 2000), and the cluster's distance to the Sun has been estimated to be in the 
range from 0.6-2.0 kpc (Walker 1957; van Altena \& Jones 1972; Sagar 1978; Sterzik et al. 1995; Sung et al. 2000; Loktin \& Beshenov 2001; Prisinzano et al. 2005).

The most recent median age estimate for the cluster, based on ground-based $B V I$ photometry down to $V=22 \mathrm{mag}$ (Prisinzano et al. 2005) is $2.3 \mathrm{Myr}$, i.e., well inside the range quoted by previous authors, although the same team also derived a much younger median age of 0.8 Myr (Damiani et al. (2004); see also Sung et al. (2000) for the pre-MS stars; Damiani et al. (2006)). Both estimates are not necessarily in conflict with each other, because the cluster appears to host stars formed over a significant length of time. While van den Ancker et al. (1997) argued that star formation in the cluster may have been ongoing since a few $\times 10^{7}$ ago, until the present, Sung et al. (2000) - based on their detailed study of low-mass PMS stars - argue that the time-scale for star formation in NGC 6530 must have been much shorter, starting only a few $\times 10^{6}$ yr ago. Nevertheless, this clearly implies that the cluster environment has been an active star-forming environment for a significant period (see also Sung et al. (2000)).

More specifically, Damiani et al. (2006) conclude, based on a detailed analysis of the distribution of optical/infrared-excess stars throughout the region, that star formation has proceeded rather undisturbed and for a longer period in the northern cluster region than near the cluster center. This is in support of suggestions by Lada et al. (1976) and Damiani et al. (2006) that the star-formation activity may have proceeded from north to south in the cluster region, which is further supported by a spatial age segregation analysis by Prisinzano et al. (2005).

The most recent distance estimate to NGC 6530, based on BVI photometry, was derived by Prisinzano et al. (2005), $D=1250 \mathrm{pc}$, corresponding to a distance modulus $(m-M)_{V, 0} \simeq 10.48 \mathrm{mag}$. This is towards the bottom end of the range in possible distances quoted by a variety of authors, $1300 \lesssim D \lesssim 2000$ pc (Prisinzano et al. (2005), their table 1 ), generally obtained photometrically from the brightest cluster member stars. In contrast, it is significantly larger than the distance obtained from Hipparcos parallaxes of seven member stars, $D=560-711$ pc (Loktin \& Beshenov 2001). In other recent studies, both van den Ancker et al. (1997) and Sung et al. (2000) determined a best distance estimate to NGC 6530 of $D=1.8 \pm 0.1 \mathrm{kpc}\left[(m-M)_{V, 0}=11.25 \pm 0.1 \mathrm{mag}\right.$, based on optical photometry and a detailed consideration of the extinction across the cluster (see also McCall et al. $(1990))$. In this paper, we opt to use the Prisinzano et al. (2005) distance determination, $D=1250$ pc.

Following the WEBDA compilation of cluster parameters, we adopt a mean reddening $E(B-V)=0.35 \mathrm{mag}$ (Sung et al. (2000); see also Chini \& Neckel (1981)), in addition to a foreground reddening of $E(B-V)=0.17$ to $0.20 \mathrm{mag}$ (McCall et al. 1990; 
van den Ancker et al. 1997; Sung et al. 2000; Prisinzano et al. 2005). There is significant variation in reddening across the NGC 6530 region, as is evident from both the distribution of stars in color-magnitude space and from the spatial variation of stellar density (Damiani et al. (2004), and references therein). In fact, the amount of differential reddening ranges from $E(B-V)=0.25$ to $0.50 \mathrm{mag}$ across the cluster (Sung et al. (2000); also Sagar (1978), but see van den Ancker et al. (1997)), although Damiani et al. (2004) also uncovered evidence for the presence of a large number of heavily reddened objects, characterized by $A_{V} \lesssim 20$ mag.

van Altena \& Jones (1972) were the first to determine the membership probabilities of 363 stars in a $60^{\prime} \times 32^{\prime}$ region centered on NGC 6530 from relative proper motion data with a time baseline of 34 years. They found 76 stars with membership probabilities, $p$, greater than 0.5 with $V \leq 13.6$ mag. More recent studies resulted in firm membership determinations for 451 stars with $p \geq 0.9$ (Zhao et al. 2006), and 237 stars (Prisinzano et al. 2007), the latter study based on spectroscopic observations around the lithium and $\mathrm{H} \alpha$ lines. Damiani et al. (2006) has suggested that the cluster may contain $\geq 1100$ members.

It should be noted that a reliable stellar membership determination is crucial for resolved star cluster studies. The Shanghai Astronomical Observatory has in its archives wide-field photographic plates with a long time baseline of these two open clusters, thus providing valuable, independent observational materials for high-accuracy measurements of stellar proper motions, and for the determination of cluster membership. This constitutes a strong starting point for further detailed cluster studies, including those dealing with the effects of mass segregation, as we show in this paper.

In this paper, we use photographic plate material with longer time baselines or higher quality than published previously, in order to determine the proper motions and membership probabilities of stars in the regions of both clusters (Sect. 2). We present a new, detailed investigation of these two clusters based on our membership determination (Sect. [3), of their stellar luminosity functions and the observed effects of mass segregation (Sect. 4). In Sect. 5 we discuss the clusters in the context of their evolutionary state and summarize the main results obtained in this paper.

\section{Observations}

For the open clusters NGC 2244 and NGC 6530, all photographic plates used for the proper motion reduction were taken by the 40-cm refractor telescope (with a focal length of $6895 \mathrm{~mm}$ ) at the Sheshan (Zô-Sè) Station of the Shanghai Astronomical Observatory, 
operated by the Chinese Academy of Sciences. For NGC 2244, four plates for each of the first and second epochs were taken in 1963-1964, and 1998-1999, respectively, spanning a time baseline of 35 years. For NGC 6530, two first epoch plates and four second plates were taken in 1912 and 1999, respectively, spanning 87 years. The field of view achieved is $2^{\circ} \times 2.5^{\circ}$ and $1.5^{\circ} \times 1.5^{\circ}$, for the first and second epoch plates, respectively. No filter was used to obtain these plates; their sensitive wavelength region is close to the $B$ band and the $30 \mathrm{~min}$ exposure time resulted in a brightness limit of about $16 \mathrm{mag}$.

The stellar coordinates on the plates of both clusters were measured with the Photometrics Data Systems (PDS) microdensitometer (Stilburn et al. 1992) at the Dominion Astrophysical Observatory (DAO) in Canada. For each plate, raster scanning was performed over the full $60^{\prime} \times 60^{\prime}$ central regions of the clusters. The scanning diaphragm used had a size of $20.5 \mu \mathrm{m}$ squared, with both a step length and line separation of $20 \mu \mathrm{m}$; a speed of $15 \mathrm{~mm}$ $\mathrm{s}^{-1}$ was used. In the region where we performed the full scan, stellar images were extracted and their rectangular coordinates, $(x, y)$, obtained using the processing software provided by DAO. After the preliminary identification, the measured coordinates on each plate were transformed linearly to a unified system using six plate constants, and those with residual errors $\geq 15 \mu \mathrm{m}$ were excluded. The cross-identification resulted in a final set of 498 stars for NGC 2244 and 365 stars for NGC 6530, respectively, with each star appearing on at least one plate of both the early and later epochs.

\section{Proper motion reduction and membership estimates}

In the reduction of our proper motion data, an iterative central-overlap technique (Russell 1976; Wang et al. 1996) was used. For NGC 2244, 78 Tycho-2 stars were selected as reference stars; their reference positions and proper motions were adopted based on the Tycho-2 Catalogue (Høg et al. 2000). During the iterative solution, three stars with extraordinarily large residual errors were removed. The final solution provided us with robust position and proper motion data of 495 stars in the cluster region. Similarly, 79 Tycho-2 catalogue stars were used as reference stars for NGC 6530, and only 1 star with an extremely large residual error in the iterative solution was removed. This resulted in a total of 364 stars with final position and proper motion values. The internal levels of uncertainty on the position and proper motion of the individual stars are given in Table 1.

Estimating the membership probability of the observed cluster stars involves a careful assessment of the distributions of both the proper motions and the positions. We assume that in position space the surface number density of the field stars follows a uniform distribution, $\psi_{\mathrm{f}}$, while the cluster members approximately follow a Gaussian profile (see, e.g., Wen et al. 
(2006), for a preliminary analysis of the stars in the NGC 6530 region with membership probabilities $p \geq 0.9), \psi_{\mathrm{c}}$, with a central density, $n_{0}$, and a dispersion, $\alpha$. Then,

$$
\psi_{\mathrm{f}}=n_{\mathrm{f}}, \quad \psi_{\mathrm{c}}\left(r_{i}\right)=n_{0} \exp \left(-\frac{r_{i}^{2}}{2 \alpha^{2}}\right),
$$

where $r_{i}$ denotes the distance of the $i^{\text {th }}$ star to the cluster center. Let $\Psi_{\mathrm{f}}$ and $\Psi_{\mathrm{c}}$ be the normalized $\psi_{\mathrm{f}}$ and $\psi_{\mathrm{c}}$, respectively, and $g=n_{0} / n_{\mathrm{f}}$. Then we have $\Psi_{\mathrm{f}}+\Psi_{\mathrm{c}}=1$ (by definition), and

$$
\begin{aligned}
& \Psi_{\mathrm{f}}\left(r_{i}\right)=\frac{1}{1+g \exp ^{-1}\left(-\frac{r_{i}^{2}}{2 \sigma_{\alpha}^{2}}\right)}, \\
& \Psi_{c}\left(r_{i}\right)=\frac{1}{1+g^{-1} \exp \left(-\frac{r_{i}^{2}}{2 \sigma_{\alpha}^{2}}\right)}
\end{aligned}
$$

Both the field stars and the cluster members have independent proper motion and position distributions. We assume that the field star proper motion is characterized by an elliptical Gaussian distribution, and that of the cluster stars is a circular Gaussian function. The overall distribution function for the field stars, $\Phi_{\mathrm{f}}$, and for the cluster stars, $\Phi_{\mathrm{c}}$, can then be written as:

$$
\begin{aligned}
\Phi_{\mathrm{f}}= & \Psi_{\mathrm{f}}\left(r_{i}\right) \times \frac{1}{2 \pi\left(1-\rho^{2}\right)^{1 / 2}\left(\sigma_{x 0}^{2}+\varepsilon_{x i}^{2}\right)^{1 / 2}\left(\sigma_{y 0}^{2}+\varepsilon_{y i}^{2}\right)^{1 / 2}} \\
& \times \exp \left\{-\frac{1}{2\left(1-\rho^{2}\right)}\left[\frac{\left(\mu_{x i}-\mu_{x \mathrm{f}}\right)^{2}}{\sigma_{x 0}^{2}+\varepsilon_{x i}^{2}}\right.\right. \\
& \left.\left.-\frac{2 \rho\left(\mu_{x i}-\mu_{x \mathrm{f}}\right)\left(\mu_{x i}-\mu_{x \mathrm{f}}\right)}{\left(\sigma_{x 0}^{2}+\epsilon_{x i}^{2}\right)^{1 / 2}\left(\sigma_{y 0}^{2}+\epsilon_{y i}^{2}\right)^{1 / 2}}+\frac{\left(\mu_{y i}-\mu_{y \mathrm{f}}\right)^{2}}{\sigma_{y 0}^{2}+\varepsilon_{y i}^{2}}\right]\right\},
\end{aligned}
$$

and

$$
\begin{aligned}
\Phi_{\mathrm{c}}= & \Psi_{\mathrm{c}}\left(r_{i}\right) \times \frac{1}{2 \pi\left(\sigma_{0}^{2}+\varepsilon_{x i}^{2}\right)^{1 / 2}\left(\sigma_{0}^{2}+\varepsilon_{y i}^{2}\right)^{1 / 2}} \\
& \times \exp \left\{-\frac{1}{2}\left[\frac{\left(\mu_{x i}-\mu_{x \mathrm{c}}\right)^{2}}{\sigma_{0}^{2}+\varepsilon_{x i}^{2}}+\frac{\left(\mu_{y i}-\mu_{y c}\right)^{2}}{\sigma_{0}^{2}+\varepsilon_{y i}^{2}}\right]\right\}
\end{aligned}
$$

Here, $\left(\mu_{x i}, \mu_{y i}\right)$ is the reduced proper motion of the $i^{\text {th }}$ star and $\left(\varepsilon_{x i}, \varepsilon_{y i}\right)$ are the estimated observational errors. $\left(\mu_{x \mathrm{f}}, \mu_{y \mathrm{f}}\right)$ and $\left(\mu_{x \mathrm{c}}, \mu_{y \mathrm{c}}\right)$ are the distribution centers of the field stars and cluster members in the vector point diagram (VPD), respectively, whereas $\left(\sigma_{x 0}, \sigma_{y 0}\right)$ and $\sigma_{0}$ are the intrinsic proper motion dispersions of the field stars and the cluster members respectively, and $\rho$ is the correlation coefficient. Together with the parameters $\alpha$ and $g$, there are 10 parameters awaiting determination. These parameters were estimated by means of a 
maximum likelihood method. When searching for the maximum of the likelihood function, a bipartition algorithm was adopted (Wang et al. 1997), and the (internal) uncertainty of the parameter estimation was evaluated based on the second derivatives of the likelihood function (Zhao \& He 1987). In order to get a handle on the external uncertainty of our proper motion determinations, we compared our derived proper motion values to those derived from the Tycho-2 Catalogue, for the 78 stars in NGC 2244 and the 79 stars in NGC 6530 in common

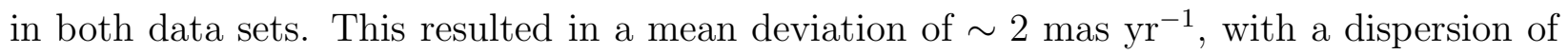
$\sim 3 \mathrm{mas} \mathrm{yr}^{-1}$, in very good agreement with the internal level of accuracy of the Tycho- 2 Catalogue.

After the distribution parameters are determined, the membership probability of the $i^{\text {th }}$ star can be calculated as:

$$
p_{i}=\frac{\Phi_{\mathrm{c}}(i)}{\Phi(i)}=\frac{\Phi_{\mathrm{c}}(i)}{\Phi_{\mathrm{f}}(i)+\Phi_{\mathrm{c}}(i)}
$$

Table 2 gives the maximum likelihood estimates of the distribution parameters, and the corresponding uncertainties, for both clusters. We used the distribution parameters to calculate the membership probabilities of the individual stars in the NGC 2244 and NGC 6530 regions. Figure 1 shows the VPD for both NGC 2244 and NGC 6530 (see also the preliminary analysis 1 by Wen et al. (2006); the solid points represent the stars with membership probabilities, $p \geq 0.9$, whereas the crosses indicate the $p \leq 0.5$. The open circles have $p$ values in between these boundaries. The VPDs already show that our membership determination appears to be quite effective. To underscore this point, Fig. 2 shows the histograms of the membership probabilities; the left-hand panel is for NGC 2244 and the right-hand panel for NGC 6530. Returning to the clusters' VPDs, both Sabogal-Martínez et al. (2001) and Higuera et al. (2002) published VPDs for NGC 2244 based on independent proper motion analyses. Although their samples of cluster members are much smaller than ours (of order 100 member stars), the VPDs are qualitatively similar in extent as well as morphology. We note, however, that in particular the Higuera et al. (2002) VPD appears somewhat more elongated in the $y$ direction than our VPD in Fig. 1. We will return to this issue below.

From Fig. 2, one can deduce quantitatively that the discrimination of membership for NGC 2244 is quite effective. Of the total of 495 stars, about $16 \%$, or 78 stars, have probabilities in the range of $0.3<p<0.7$. As an aside, we note that a comparison of

\footnotetext{
${ }^{1}$ We note that whereas Wen et al. (2006) published a preliminary analysis based on the photographic plates also used in the present paper, we emphasize that the results in the present paper are based on a thorough and significantly improved re-analysis of the entire data set (e.g., in terms of the statistical treatment of the uncertainties and membership probabilities, as well as the determination of the basic cluster parameters).
} 
the membership histograms in Sabogal-Martínez et al. (2001) and Fig. 2 shows that our membership determination is more efficient than theirs. Meanwhile, for NGC 6530, the separation of members from the field stars is even more effective, with only a few stars having probabilities around $p=0.5$, i.e., only $4.4 \%$ (or 16 stars) have $0.3<p<0.7 ; 451$ stars have $p \geq 0.9$ (see also Zhao et al. (2006) for a discussion of the results in relation to the earlier work by van Altena \& Jones (1972)). We note that in the context of NGC 6530, the Prisinzano et al. (2005, 2007) and Damiani et al. (2006) studies cover a much smaller field of view (diameter $\sim 20 \mathrm{arcmin}$ ) than our observations presented here (Sect. 2), although at a higher spatial resolution. Secondly, the Prisinzano et al. (2007) sample, which - in essence - only covers the cluster core, is fainter $(B \gtrsim 15 \mathrm{mag})$ than ours $(B \lesssim 14 \mathrm{mag})$. As such there is virtually no overlap between both data sets.

\section{Luminosity functions and the effects of mass segregation}

\subsection{Star counts and the cluster sizes}

Star counts allow us to statistically determine the properties of clusters with respect to the surrounding stellar background. The cluster radius itself is one of the most important cluster parameters, and particularly useful for dynamical studies. From our results, the member stars of both NGC 2244 and NGC 6530 show an obvious concentration on the sky, while this is clearly not the case for field stars. In essence, this supports the result for NGC 2244 by Sabogal-Martínez et al. (2001) based on the cluster's VPD. They determined robust membership probabilities for 92 stars in the area using both the cluster's VPD and its membership probability histogram, akin to our Fig. 2. In the VPD they clearly showed the (elliptical) distribution of the proper motions of the cluster members around a centroid, and the very small dispersion of stars with $p \geq 0.7$, clearly setting them apart from the field stars, with $p \lesssim 0.6$ (see also Higuera et al. (2002)). As regards NGC 6530, Wen et al. (2006) and Zhao et al. (2006) provided compelling evidence for the superiority of the membership determinations in the present data set compared to that presented by van Altena \& Jones (1972).

In order to derive the radial stellar surface density, we first chose the cluster center as the median coordinates of all the member stars with $p \geq 0.9$. The adopted centers are at the (J2000.0) equatorial coordinates of $\left(06^{\mathrm{h}} 32^{\mathrm{m}} 04^{\mathrm{s}},+4^{\circ} 55^{\prime} 00^{\prime \prime}\right)$ for NGC 2244 and $\left(18^{\mathrm{h}} 04^{\mathrm{m}} 24^{\mathrm{s}},-24^{\circ} 21^{\prime} 12^{\prime \prime}\right)$ for NGC 6530 , respectively. These updated center positions are slightly different from the values provided by WEBDA, although the displacements between our new center coordinates and those provided by WEBDA are well inside the respective cluster core radii (see below). For comparison, Li (2005) determined the 2MASS center 
position of NGC 2244 as being very close to the centroid determined from our proper motions, resulting in J2000.0 equatorial coordinates of $\left(06^{\mathrm{h}} 31^{\mathrm{m}} 59.9^{\mathrm{s}},+4^{\circ} 55^{\prime} 36^{\prime \prime}\right)$. The radial number density profile, shown in Fig. 3, was constructed by performing star counts inside increasing concentric annuli around the cluster center and normalized by the surface areas covered by the respective annuli.

Figure 3 shows the radial surface number density profiles of the cluster members $(p \geq$ $0.9)$ and the field stars $(p \leq 0.1)$ in our observational fields of view, on the left-hand side for NGC 2244 and on the right-hand side for NGC 6530. In each panel, the profiles for the cluster members and the field stars are completely different, with the field star distributions essentially flat, whereas the cluster members exhibit obvious concentrations towards the cluster centers. This result shows once again that our membership determinations are reasonably robust and reliable. The basic structural parameters were derived by fitting the two-parameter surface density King (1966) profile to our observational data in a least-squares sense. We used fitting radii of 32 and 33 arcmin for NGC 2244 and NGC 6530, respectively. The core radii thus obtained are $9.43 \pm 1.48$ arcmin for NGC 2244 and $4.29 \pm 0.90$ arcmin for NGC 6530, corresponding to $4.7 \pm 0.7$ and $1.6 \pm 0.3 \mathrm{pc}$, respectively. Meanwhile, the half-number radii (i.e., the radii on either side of which half of the member stars are located) of the clusters were also determined, as 14 arcmin for NGC 2244 and 21 arcmin for NGC 6530 , or $\sim 6.9$ and $\sim 7.6 \mathrm{pc}$, respectively. Our main reason for using the clusters' halfnumber radii instead of their half-light radii is practical: the position and proper motion information, eventually leading to membership probabilities, is based on the results from the PDS scans. During this process, some of the brightest stars had to be avoided because of saturation effects on the photographic plates (which would lead to unacceptably large positional uncertainties for our analysis). In other words, our sample of cluster members is incomplete at bright magnitudes, and the membership probabilities of the (few) brightest stars are unknown. The half-number radii are not significantly affected by the omission of these few stars, which would have an extraordinarily large contribution to the clusters' total fluxes, however.

Although Li (2005) found that the radial profile in the near-infrared of the NGC 2244 member stars disappears into the background field star population at a radius of about 20 arcmin 2, our radial profile in Fig. 3 clearly shows that we can follow the cluster's profile out to almost 30 arcmin. This is in keeping with the cluster radius of 11.8 pc derived by

\footnotetext{
${ }^{2}$ See also Li \& Smith (2005b), who claim that the total extent of the cluster is about 20 pc, which at a distance of $1.4 \mathrm{kpc}$ - translates into a linear diameter of about $49 \operatorname{arcmin}$. Similar size estimates were obtained in the optical by Townsley et al. (2003), and in X-rays by Berghöfer \& Christian (2002) and Chen et al. (2004).
} 
Sabogal-Martínez et al. (2001), which - at an assumed distance of $1.4 \mathrm{kpc}$ - corresponds to about 29 arcmin. Our size determination for NGC 6530 is less clear-cut, as Fig. 3 shows: the radial profile composed by its member stars appears to merge with the field star population at a radius of around 20 arcmin, although this value is rather uncertain. We believe, therefore, that our result is in reasonable agreement with the diameter of roughly 35 arcmin quoted by van den Ancker et al. (1997).

For both clusters, the model profiles tend to roughly follow the observational data points in the outer regions, but not in detail. This suggests that both clusters do not have welldefined outer radii, but are instead characterized by a significant amount of substructure. There is, in fact, strong evidence that NGC 2244 possesses distinct substructures. Li (2005), using 2MASS near-infrared imaging, found that the cluster is resolved into an extended distribution of stellar sources associated with its compact core in the south center of the blister HiI region, but also into a clear elongated density enhancement in the west. NGC 6530 also exhibit distinct substructures: in a region towards the northwest of the actual cluster center there appears to be a density enhancement of slightly older stars than in the rest of the cluster (e.g., Damiani et al. (2006)). In addition, the VPDs of both clusters (Fig. 1; see also Higuera et al. (2002), in particular) appear to have axial ratios significantly deviating from unity.

Finally, Li (2005) and Li \& Smith (2005b) point out that instead of using a King (1966) profile, a more appropriate radial density profile might be represented by a $R^{-1}$ profile, following suggestions by Baba et al. (2004) regarding the radial density distributions of embedded star clusters. In Fig. 3, we show the best-fitting $R^{-1}$ profiles for both clusters. For NGC 2244, the best-fitting profile follows $n(R)=0.046+0.615 R^{-1}$, while the equivalent profile for NGC 6530 is $n(R)=0.020+0.791 R^{-1}$. We note that whereas the fit to the NGC 6530 radial profile is statistically good, the NGC 2244 distribution is better approximated by a King profile.

\subsection{Luminosity distributions}

Massey et al. (1995) provided their $U B V$ observations centered on NGC 2244 with the KPNO $0.9 \mathrm{~m}$ telescope and a Tektronix $2048 \times 2048 \mathrm{CCD}$, covering a $2800 \mathrm{arcmin}^{2}$ area. This resulted in accurate photometric data for a total of 773 stars in the cluster region, down to $B \approx 18$ mag. By matching our photographic plate observations to the Massey et al. (1995) sample using a conservative matching tolerance of 0.3 arcsec, 323 stars in common were identified. Furthermore, using a quadratic function, our initial instrumental magnitudes, $B_{p}$, were converted to the $B$ magnitudes in the Massey et al. (1995) system. 
As regards NGC 6530, Prisinzano et al. (2005) performed BVI observations of the NGC 6530 region using the ESO $2.2 \mathrm{~m}$ telescope at La Silla, characterized by a field of view of $34 \times 33 \operatorname{arcmin}^{2}$. The limiting magnitude of their work is $V \approx 17.5$ mag. We compared our $B_{p}$ instrumental magnitudes with the $B$ magnitudes of 62 stars in common with Prisinzano et al. (2005)'s sample. Except for a few stars, we found an obvious linear correlation between the magnitudes, which we used to convert our $B_{p}$ photometry to the B magnitudes in the $U B V$ system.

Figure 4 shows the comparison between our instrumental $B_{p}$ photometry and the $B$ magnitudes of $U B V$ system, for both clusters. The photometric uncertainties in both our own data and those taken from the literature are small: Massey et al. (1995) state that their uncertainties are $<0.02 \mathrm{mag}$ for stars brighter than $V=14.5 \mathrm{mag}$, and "do not become appreciable until $V=16$ mag". The photometric uncertainties in the Prisinzano et al. (2005) data have a mean value of $0.02 \mathrm{mag}$, with a maximum error $<0.2 \mathrm{mag}$.

For member stars in a given open cluster, all at roughly the same distance, the range in visual magnitudes corresponds to that in luminosity. In addition, when only main-sequence stars are considered, the stellar luminosity is a proxy for the stellar mass. In order to assess the effects of mass segregation, we first examined the luminosity functions of the member stars in different (radial) ranges of the clusters. Figures 5 and 6 show the $M_{B}$ luminosity functions of stars with membership probabilities $p \geq 0.9$ in the two cluster regions.

Figures 5a (top) and b (middle) show the luminosity functions of the NGC 2244 cluster members in the inner $\left(R \leq R_{\mathrm{h}}\right)$ and outer $\left(R>R_{\mathrm{h}}\right)$ areas of NGC 2244, where $R_{\mathrm{h}}=14$ arcmin is the half-number radius derived from our full sample of 216 member stars. Fig. 5c (bottom) shows the cumulative distributions for both radial regions.

Figures 6a (top), b (middle), and c (bottom) show the equivalent results for NGC 6530 (see also Zhao et al. (2006)). Here, we derived a half-number radius of $R_{\mathrm{h}}=21$ arcmin from our total sample of 250 member stars.

From these two figures, the effects of mass segregation are evident: the relative fraction of brighter stars (or, equivalently, more massive stars) is higher in the inner than in the outer regions. As an alternative approach to investigate these mass segregation effects, we calculated the half-number radii for member stars in different absolute magnitude ranges, i.e., for $M_{B} \leq 0$ and $M_{B}>0$ mag. The results of this exercise are listed in Table 5 (see Zhao et al. (2006) for a complementary discussion related to NGC 6530; in particular their table 6). It can be seen that for both clusters, the brighter members are characterized by smaller half-number radii - another indication of the existence of mass segregation in these clusters. 
To further examine the mass segregation effects, we also investigated the radial density distributions of member stars $(p \geq 0.9)$ in different luminosity ranges in the clusters, which are shown in Figs. 7 (for NGC 2244) and 8 (for NGC 6530). In each cluster, a positiondependent LF is found. In essence, the fainter (i.e., lower-mass) stars are spread throughout the entire cluster in both objects, while the brighter (massive) stars are predominantly located in the inner region.

\subsection{Velocity-luminosity relation}

If the mass segregation effects solely result from the process of two-body relaxation and the associated energy equipartition, one should find some evidence for this effect not only in the spatial distribution of the cluster members, but also in their velocity distribution: the more massive members will have a smaller velocity dispersion than the less massive stars.

Accordingly, for true cluster members (with $p \geq 0.95$ ) in different magnitude ranges, the intrinsic proper motion dispersions $\sigma_{\text {int }}$ as derived from the observed dispersions $\sigma_{\text {obs }}$, and the mean errors intrinsic to the stellar proper motions were investigated. The final results are shown in Figs. 9 and 10. It can be seen from these figures that the intrinsic proper motion dispersions are not significantly different for various magnitude groups. Thus, our data do not support any significant velocity (dispersion)-mass correlation, neither in NGC 2244, nor in NGC 6530 (see also Zhao et al. (2006)).

\section{Discussion and Summary}

The traditional explanation for (position dependent) mass segregation in an open cluster is that it results from a situation in which all cluster stars approach energy equipartition. However, this is unlikely the case for NGC 2244 and NGC 6530, since both clusters are very young, low-density objects (but see Portegies Zwart et al. (2007) for counterarguments). With ages of only around 2-3 Myr, their ages are comparable to the time-scale of about only one or two crossing times.

Theoretically, the nature and degree of "primordial" mass segregation, i.e., mass segregation intrinsic to the star-formation process itself, is presumably determined by the properties of the interactions of protostellar material during the star-forming episode in a cluster. More massive stars are subject to more mergers, hence accrete even more mass (cf. Larson (1991); Bonnell et al. (2001a,b) and references therein), and therefore dissipate more kinetic energy. In addition, they tend to form near the cluster center, in the highest-density re- 
gion, where the encounter-rate is highest (Larson 1991; Bonnell et al. 1997, 1998, 2001a,b; Bonnell \& Davies 1998). This will lead to an observed position-dependent stellar mass function containing more low-mass stars at larger radii compared to the mass function in the cluster center (although low-mass stars are still present at small radii, see also the discussions in de Grijs et al. (2002a, b) ; but see Portegies Zwart et al. (2007) for arguments in favor of excluding primordial mass segregation to explain recent observations of the Arches cluster near the Galactic Center).

From an observational point of view, the study of very young star clusters still embedded in the molecular clouds from which they originated might give us a handle to constrain the degree of primordial mass segregation. In the Milky Way, in three such young star clusters mass segregation effects have been studied in great detail in the past two decades.

While Lada et al. (1991) suggested, based on ground-based observations, that the brighter stars in NGC 2024 (and the Mon R2 complex at large) seem to be more centrally concentrated than the fainter cluster members, this evidence was deemed inconclusive by Carpenter et al. (1997). They argued that this result was based on an incomplete sample of cluster members, although mass segregation might be limited to the massive, bright OB stars forming in the very center. These same authors argued that for masses below $2 M_{\odot}$, mass segregation effects in the Mon R2 complex amount to only a $\sim 2 \sigma$ result.

Secondly, a combination of both ground-based (e.g., Hillenbrand (1997) and Hubble Space Telescope (HST) observations (e.g., Hillenbrand \& Hartmann (1998)) of the Orion Nebula Cluster (ONC), and in particular of its very core, the Trapezium stars, have presented clear evidence for mass segregation for the $m>5 M_{\odot}$ component, with some evidence for general mass segregation down to $m \simeq 1-2 M_{\odot}$ (Hillenbrand \& Hartmann (1998); see also the review by Larson (1993)).

Finally, R136, the central cluster in 30 Doradus in the Large Magellanic Cloud (LMC; age $\lesssim 3-4$ Myr; cf. Hunter et al. (1995)), has been studied extensively, both from the ground and with the HST. A variety of techniques have revealed a significant overabundance of highmass stars in its very center, thus supporting strong mass segregation (e.g., Cambell et al. (1992); Larson (1993); Malumuth \& Heap (1994); Brandl et al. (1996)).

Thus, in most of the (still partially embedded) young star clusters that can be resolved in individual stars, mass segregation effects are observed, although to varying degrees. This underlines the importance of our understanding of the physical processes involved in the formation and evolution of star clusters, and in particular of the IMF, which will ultimately determine the time-scale on which a young star cluster will eventually be destroyed.

We may conclude from the above discussion that the observed spatial mass segregation 
in the young open clusters NGC 2244 and NGC 6530 might have resulted from a combination of both initial conditions in the early stages of evolution and, possibly, two-body relaxation process. The latter process will lead to a manifestation of cluster-wide mass segregation on the half-mass relaxation time-scale. However, it will proceed much faster in higher-density regions in the cluster, and among the more massive stars - both of these conditions favor an decreased time-scale in the cluster center.

Finally, we will consider the long-term fate of the two young clusters discussed in this paper in relation to their (observed) IMF and velocity dispersion. Both clusters have been scrutinized with the aim of determining their stellar make-up. Because of their very young ages, the stellar content in essence reflects the mass distribution at the time of their birth, as insufficient time has passed for any significant number of stars to have undergone a full cycle of stellar evolution (and neither for significant dynamical evolution to have occurred, as argued above).

Park \& Sung (2002) determined the IMF of NGC 2244 in the mass range $-0.5 \leq$ $\log \left(m_{*} / M_{\odot}\right) \leq 2.0$. They found a flat IMF slope, with $\Gamma=-0.7 \pm 0.1$, where the equivalent slope of the Salpeter (1955) IMF would be $\Gamma=-1.35$. Their result is in close agreement both with that of Massey et al. (1995) in the range from $\sim 7$ to $15 M_{\odot}$, and with that of Pérez (1991) for stars with masses in excess of $4 M_{\odot}, \Gamma=-0.7$.

Such a flat IMF implies that the cluster's stellar population contains too many massive stars with respect to their lower-mass counterparts for it to survive the dynamical effects leading to cluster dissolution for any significant amount of time (see, e.g., Smith \& Gallagher (2001) in the context of the young massive cluster M82-F; and Chernoff \& Shapiro (1987); Chernoff \& Weinberg (1990); Goodwin (1997) for theoretical arguments in support of this notion). The precise dissolution time-scale depends sensitively on the IMF mass range (Kouwenhoven et al., in prep.). This conclusion is supported by arguments related to the cluster's velocity dispersion. Its intrinsic overall velocity dispersion is $\sigma_{\text {tot }}^{\text {int }}=4.45 \pm 0.15$ mas $\mathrm{yr}^{-1}$; at the adopted distance, $D=1.7 \mathrm{kpc}$, this is equivalent to $\sigma_{\mathrm{tot}}^{\mathrm{int}} \simeq 35 \mathrm{~km} \mathrm{~s}^{-1}$. 3 This large linear velocity dispersion is significantly in excess of the "typical" velocity dispersions older open clusters in the Milky Way characterized by a Salpeter-type IMF, or equivalent (de Grijs et al., in prep.). Thus, the combination of these arguments leads us to conclude that NGC 2244 will most likely dissolve on a short time-scale (see also Li \& Smith (2005b) for a more conservative back-of-the-envelope calculation supporting our conclusion). - if not due

\footnotetext{
${ }^{3}$ We note that if we assume that this is the cluster's expansion velocity, the dynamical age of the starforming region centered on the cluster is of order 0.4 Myr. This is remarkably close to the dynamical age of the nebula, estimated at $0.2-0.6$ Myr by Matthews (1967).
} 
to internal two-body relaxation effects, then most likely because of external shocks expected to operate near its location in, or close to, the Galactic disk (although we note that these effects may not be very significant in the Galactic anticenter direction)

NGC 6530, on the other hand, exhibits a power-law IMF slope of $\Gamma=-1.22 \pm 0.17$ for stellar masses between 0.6 and $4 M_{\odot}$ (Prisinzano et al. 2005), fully consistent with the Salpeter (1955) IMF. They note that the cluster IMF peaks towards smaller masses and then declines. This is in keeping with the detailed photometric study of Sung et al. (2000), who quote an IMF slope of $\Gamma=-1.3 \pm 0.1$, largely independent of the mass to luminosity conversion employed. Its intrinsic velocity dispersion is $\sigma_{\text {tot }}^{\text {int }}=1.48 \pm 0.14{\text { mas } \mathrm{yr}^{-1}}$; at the adopted distance, $D=1250 \mathrm{pc}$, this is equivalent to $\sigma_{\text {tot }}^{\text {int }} \simeq 8 \mathrm{~km} \mathrm{~s}^{-1}$, i.e., much closer to the dynamical state required for longer-term survival, although still fairly large (de Grijs et al., in prep.). In the absence of sizeable external perturbations, we speculate, therefore, that NGC 6530 has the potential to survive for a significant length of time, possibly of order a few $\times 10^{8}$ yr. However, we also note that the cluster is situated very close to the Galactic plane in the direction of the Galactic Center, such that external perturbations are unlikely to remain insignificant for any length of time.

Finally, we point out that the large velocity dispersions obtained for both young clusters imply that these objects are unlikely to survive for any significant length of time. The velocity dispersions of 'classical' old ( $\gtrsim 10^{8} \mathrm{yr}$ ) bound open clusters are of order $\lesssim 1.5 \mathrm{~km}$ $\mathrm{s}^{-1}$ as shown by, e.g., Lohman (1972) in what is probably the most comprehensive study of open cluster velocity dispersions available to date. A small subset of these measurements have since been confirmed in more recent studies of individual objects (de Grijs et al., in prep.). For instance, the velocity dispersion of the $522 \pm 82$ Myr-old (Paunzen \& Netopil 2006) central cluster in Coma Berenices is $\sigma_{1 \mathrm{D}}=0.27 \pm 0.07 \mathrm{~km} \mathrm{~s}^{-1}$ (Lohman (1972); cf. $\sigma_{1 \mathrm{D}} \sim 0.3 \mathrm{~km} \mathrm{~s}^{-1}$ measured in the cluster core by Odenkirchen et al. (1998)); that of M67 (at an age of $\sim 4.0 \mathrm{Gyr}$; Paunzen \& Netopil (2006)) ranges from $0.49-1.28 \mathrm{~km} \mathrm{~s}^{-1}$, with a best estimate of $0.81 \pm 0.10 \mathrm{~km} \mathrm{~s}^{-1}$ (see Girard et al. 1989, de Grijs et al., in prep.), while the velocity dispersion of Praesepe (M44) is $0.46 \pm 0.2 \mathrm{~km} \mathrm{~s}^{-1}$ (Jones 1971), at an age of $753 \pm 201$ Myr (Paunzen \& Netopil 2006). M35 $\left(\log (\right.$ Age/yr $)=8.26_{-0.30}^{+0.05}$; Kalirai et al. $(2003))$ is characterized by $\sigma_{1 \mathrm{D}}$ from $0.76 \pm 0.19 \mathrm{~km} \mathrm{~s}^{-1}$ (Lohman 1972) to $1.00 \pm 0.10 \mathrm{~km}$ $\mathrm{s}^{-1}$ (McNamara \& Sekiguchi 1986). Although we refer the reader for further details on the kinematic structure and long-term stability of Galactic open clusters to a forthcoming paper (de Grijs et al., in prep.), these examples show that in order for an open cluster to survive for a few $\times 10^{8} \mathrm{yr}$, its velocity dispersion needs to be sufficiently small so as to prevent early dissolution of the cluster due to internal kinematic effects. Neither of the clusters discussed in this paper satisfies this empirical condition. The only old $(\log$ (Age/yr) $=8.4 \pm 0.1$; Sung et al. (1999)) Galactic open cluster with a measured velocity dispersion in excess of 
the range indicated above is M11, with $\sigma_{1 \mathrm{D}}$ from $1.21 \pm 0.35 \mathrm{~km} \mathrm{~s}^{-1}$ (Mathieu 1984) to 2.9 $\mathrm{km} \mathrm{s}^{-1}$ (McNamara \& Sanders 1977); the latter measurement was later reduced to $2.0 \mathrm{~km}$ $\mathrm{s}^{-1}$ (McNamara \& Sekiguchi 1986). This high velocity dispersion, for an ostensibly stable massive open cluster, is still more than a factor of two below that of NGC 6530, which is both significantly less massive and less compact than M11, and thus less stable to kinematic dissolution.

The main points of the present study can be summarized as follows:

1. From the photographic plate data of the Shanghai Astronomical Observatory, the proper motions of stars in the region of the open clusters NGC 2244 and NGC 6530 were reduced by means of a central-overlapping technique, and the distribution parameters of the clusters as well as the membership probabilities of the individual stars in the cluster region were determined using a maximum likelihood principle.

2. Both clusters show clear evidence of mass segregation, but there is no definite evidence for a velocity (dispersion)-mass (or, equivalently, a velocity-luminosity) dependence in these clusters. The observed mass segregation might be due to a combination of both initial conditions and relaxation processes.

3. Based on arguments related to the clusters' IMFs, in conjunction with our new measurements of their internal velocity dispersions, we provide strong arguments in favor of the dissolution of NGC 2244 on very short time-scales, while we speculate that NGC 6530 may be more stable against the effects of internal two-body relaxation.

RdG was partially supported by an "International Joint Project" grant, jointly funded by the Royal Society in the UK and the Chinese National Science Foundation (NSFC). LC \& JLZ want to thank Dr. Peter Stetson of DAO for his kind help in measuring plates with the PDS machine. LC and JLZ are supported by the NSFC (Grant Nos. 10333050, 10333020). This research has made use of the WEBDA database, operated at the Institute for Astronomy of the University of Vienna.

\section{REFERENCES}

Baba, D., et al. 2004, ApJ, 614, 818

Berghöfer,T. W., \& Christian, D. J. 2002, A\&A, 384, 890 
Blitz, L., \& Stark,A. A. 1986, ApJ, 300, L89

Bonnell, I. A., Bate, M. R., Clarke, C. J., \& Pringle,J. E. 1997, MNRAS, 285, 201

Bonnell, I. A., \& Davies, M. B. 1998, MNRAS, 295, 691

Bonnell, I. A., Bate, M. R., \& Zinnecker, H. 1998,MNRAS, 298, 93

Bonnell, I. A., Bate, M. R.,Clarke, C. J., \& Pringle, J. E. 2001a, MNRAS, 323, 785

Bonnell, I. A., Clarke, C. J., Bate, M. R., \& Pringle,J. E. 2001b, MNRAS, 324, 573

Brandl, B., Sams, B. J., Bertoldi, F., Eckart, A.,Genzel, R., Drapatz, S., Hofmann, R., Löwe, M., \& Quirrenbach, A. 1996, ApJ, 466, 254

Campbell, B., et al. 1992, AJ, 104, 1721

Carpenter, J. M., Meyer, M. R., Dougados, C., Strom,S. E., \& Hillenbrand, L. A. 1997, AJ, 114,198

Chen, W. P., Chiang, P.S. \& Li, J.Z. 2004, ChJAA, 4, 153

Chernoff, D. F., \& Shapiro, S. L. 1987, ApJ, 322, 113

Chernoff, D. F., \& Weinberg, M. D. 1990, ApJ, 351, 121

Chini, R., \& Neckel, T. 1981, A\&A, 102, 171

Cudworth, K. 1976, AJ, 81, 519

Damiani, F., et al. 2004, ApJ, 608, 781

Damiani, F., Prisinzano, L., Micela, G., \& Sciortino, S. 2006, A\&A, 459, 477

de Grijs, R., Gilmore, G. F., Johnson, R. A., \& Mackey, A. D. 2002a, MNRAS, 331, 245

de Grijs, R., Gilmore, G. F., Mackey, A. D., Wilkinson, M. I., Beaulieu, S. F., Johnson, R. A., \& Santiago, B. X. 2002b, MNRAS, 337, 597

Girard, T. M., Grundy, W. M., López, C. E., \& van Altena, W. F. 1989, AJ, 98, 227

Goodwin, S. P. 1997, MNRAS, 284, 785

Hensberge, H., Pavlovski, K., \& Verschueren, W. 2000, A\&A, 358, 553

Higuera G., M. A., Uribe, A., \& Barrera, R. S. 2002, Rev. Mex. A\&A (Ser. de Conf.), 14, 33 
Hillenbrand, L. A. 1997, AJ, 113, 1733

Hillenbrand, L. A., \& Hartmann, L. E. 1998, ApJ, 492, 540

Høg E., Fabricius C., Makarov V.V., Urban S., Corbin T., Wycoff G., Bastian U., Schwekendiek P. \& Wicenec A. 2000, A\&A, 355, L27

Hunter, D. A., Shaya, E. J., Holtzman, J. A., Light, R. M., O’Neil, E. J., \& Lynds, R. 1995, ApJ, 448, 179

Jones, B. 1971, AJ, 76, 470

Kalirai, J. S., Fahlman, G. G., Richer, H. B., \& Ventura, P. 2003, AJ, 126, 1402

King, I. 1966, AJ, 71, 64

Lada, C. J., Gottlieb, C. A., Gottlieb, E. W., \& Gull, T. R. 1976, ApJ, 203, 159

Lada, E. A., DePoy, D. L., Evans, N. J., \& Gatley, I. 1991, ApJ, 371, 171

Larson, R. B. 1991, in: Fragmentation of Molecular Clouds and Star Formation, IAU Symp. 147, Falgarone, E., Boulanger, F., \& Duvert, G., eds., Dordrecht: Kluwer, p. 261

Larson, R. B. 1993, in: The Globular Cluster-Galaxy Connection, Smith, G. H., \& Brodie, J. P., eds., ASP Conf. Ser. 48, (ASP: San Francisco), p. 675

Larson, R. B. 2003, ASP Conf. Ser., 287, 65

Li, J. Z. 2005, ApJ, 625, 242

Li, J. Z., \& Smith, M. D. 2005a, A\&A, 431, 925

Li, J. Z., \& Smith, M. D. 2005b, AJ, 130, 721

Lohman, W. 1972, Astron. Nachr., 293, 259

Loktin, A. V., \& Beshenov, G. V. 2001, Astr. Lett., 27, 386

Malumuth, E. M., \& Heap, S. R. 1994, AJ, 107, 1054

Marschall, L. A., van Altena, W. F., \& Chiu, L.-T. 1982, AJ, 87, 1497

Massey, P., Johnson, K. E., \& Degioia-Eastwood, K. 1995, ApJ, 454, 151

Mathieu, R. D. 1984, ApJ, 284, 643 
Matthews, W. G. 1967, ApJ, 147, 965

McCall, M. L., Richer, M. G., \& Visvanathan, N. 1990, ApJ, 357, 502

McNamara, B. J., \& Sanders, W. L. 1977, A\&A, 54, 569

McNamara, B. J., \& Sekiguchi, K. 1986, AJ, 310, 613

Mermilliod, J.-C., \& Paunzen, E. 2003, A\&A, 410, 511

Odenkirchen, M., Soubiran, C., \& Colin, J. 1998, NewA, 3, 583

Ogura, K., \& Ishida, K. 1981, PASJ, 33, 149

Park, B.-G., \& Sung, H., 2002, AJ, 123, 892

Paunzen, E., \& Netopil, M. 2006, MNRAS, 371, 1641

Pérez, M. R., The, P. S., \& Westerlund, B. E., 1987, PASP, 99, 1050

Pérez, M. R., Jones, M. D., The, P. S., \& Westerlund, B. E. 1989, PASP, 101,195

Pérez, M. R. 1991, Rev. Mex. A\&A, 22, 99

Portegies Zwart, S. F., Gaburov, E., Chen, H.-C., \& Gürkan, M. A. 2007, MNRAS (letters), in press (astro-ph/0702693)

Prisinzano, L., Damiani, F., Micela, G., \& Sciortino, S. 2005, A\&A, 430, 941

Prisinzano, L., Damiani, F., Micela, G., \& Pillitteri, I. 2007, A\&A, 462, 123

Rauw, G., Nazé, Y., Gosset, E., Stevens, I. R., Blomme, R., Corcoran, M. F., Pittard, J. M., \& Runacres, M. C. 2002, A\&A, 395, 499

Russell, J. L. 1976, Ph.D. Thesis, University of Pittsburgh

Sabogal-Martínez, B. E., García-Varela, J. A., Higuera G., M. A., Uribe, A., \& Brieva, E. 2001, Rev. Mex. A\&A, 37, 105

Sagar, R., \& Joshi, U. C. 1978, MNRAS, 184, 467

Salpeter, E. E. 1955, ApJ, 121, 161

Smith, L. J., \& Gallagher, J. S. III 2001, MNRAS, 326, 1027

Sterzik, M. F., Alcala, J. M., Neuhäuser, R., \& Schmitt, J. H. M. M. 1995, A\&A, 297, 418 
Stilburn, J. R., Stetson, P. B., \& Fisher, W. A. 1992, JRASC, 86, 140

Sung, H., Bessell, M. S., Lee H.-W., Kang, Y. H., \& Lee, S.-W. 1999, MNRAS, 310, 982

Sung, H., Chun, M.-Y., \& Bessell, M. S. 2000, AJ, 120, 333

The, P.-S. 1960, ApJ, 132, 40

Townsley, L. K., Feigelson, E. D., Montmerle, T., Broos, P. S., Chu, Y.-H., \& Garmire, G. P. 2003, ApJ, 593, 874

Turner, D. G. 1976, ApJ, 210, 65

van Altena, W. F., \& Jones, B. F. 1972, A\&A, 20, 425

van den Ancker, M. E., The, P. S., Feinstein, A., Vázquez, R. A., de Winter, D., \& Pérez, M. R. 1997, A\&AS, 123, 63

van Schewick, H. 1958, Veroff. Univ. Sternwarte, No. 51

Verschueren, W. 1991, Ph.D. Thesis, Vrije Univ. Brussels (VUB)

Walker, M. F. 1957, ApJ, 125, 636

Wang, J. J., Chen, L., \& Zhao, J. H. 1996, Acta Astr. Sin., 37, 68

Wang, J. J. 1997, Annals of Shanghai Observatory, Acad. Sinica, 18, 45

Wen, W., Zhao, J.-L., \& Chen, L. 2006, Chin. A\&A, 30, 274

Williams, J. P., Blitz, L., \& Stark, A. A. 1995, ApJ, 451, 252

Zhao, J.-L., Chen, L., \& Wen W. 2006, ChJA\&A, 4, 435

Zhao, J. L., \& He, Y. P. 1987, Acta Astr. Sin., 28, 374 

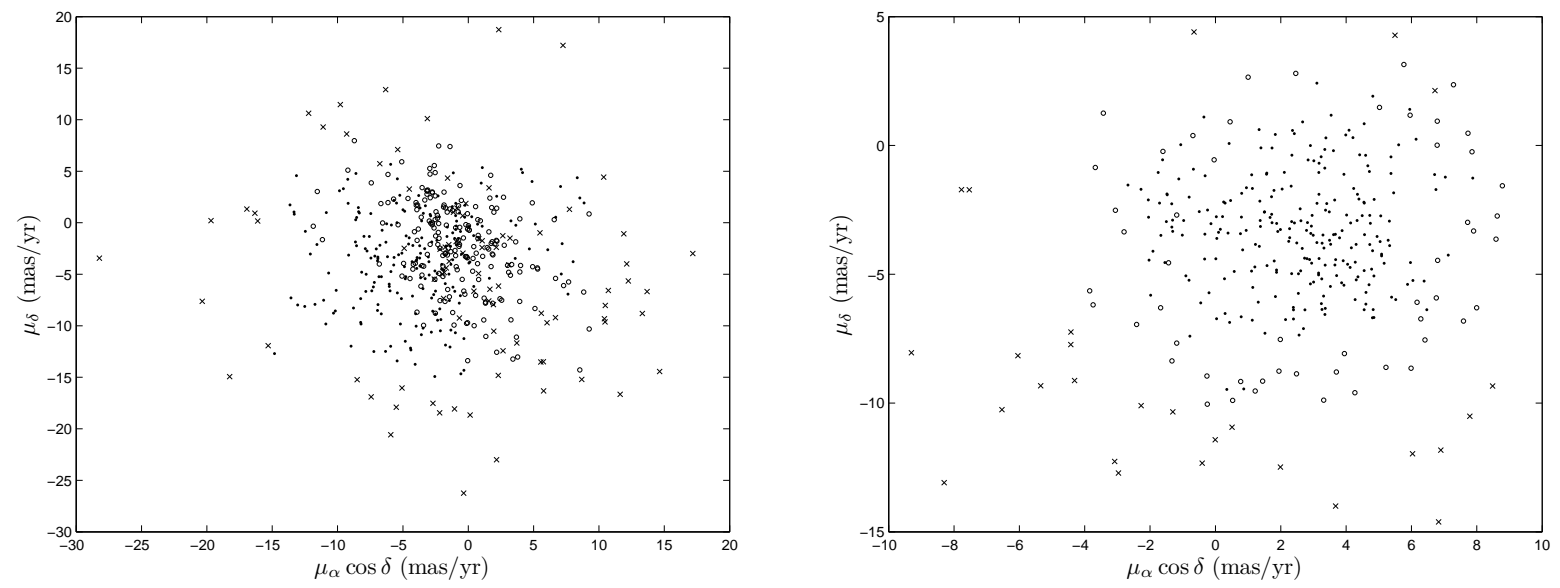

Fig. 1.- Vector-point diagrams for both NGC 2244 (left) and NGC 6530 (right); the solid points represent the stars with membership probabilities $p \geq 0.9$, whereas the crosses indicate the $p \leq 0.5$. The open circles have $p$ values in between these boundaries.
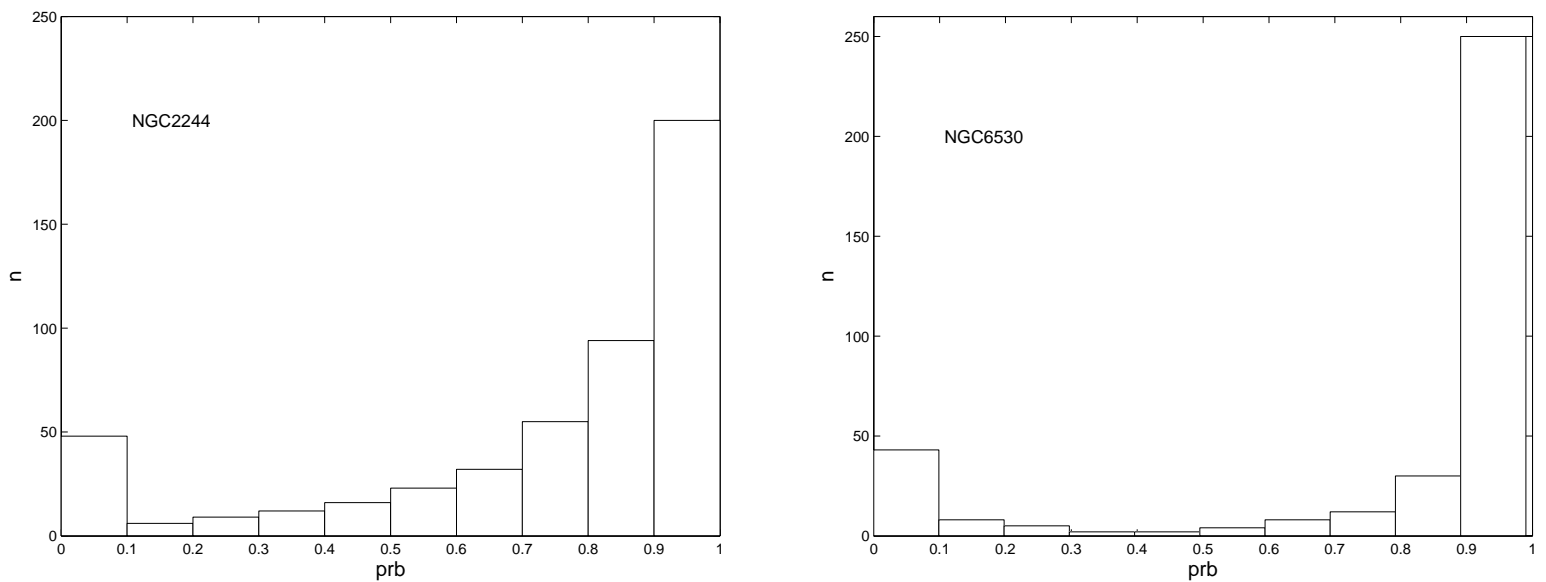

Fig. 2.- Histogram of the stellar membership probabilities. Left panel: 495 stars in the region of NGC 2244; Right panel: 364 stars in region of NGC 6530. 

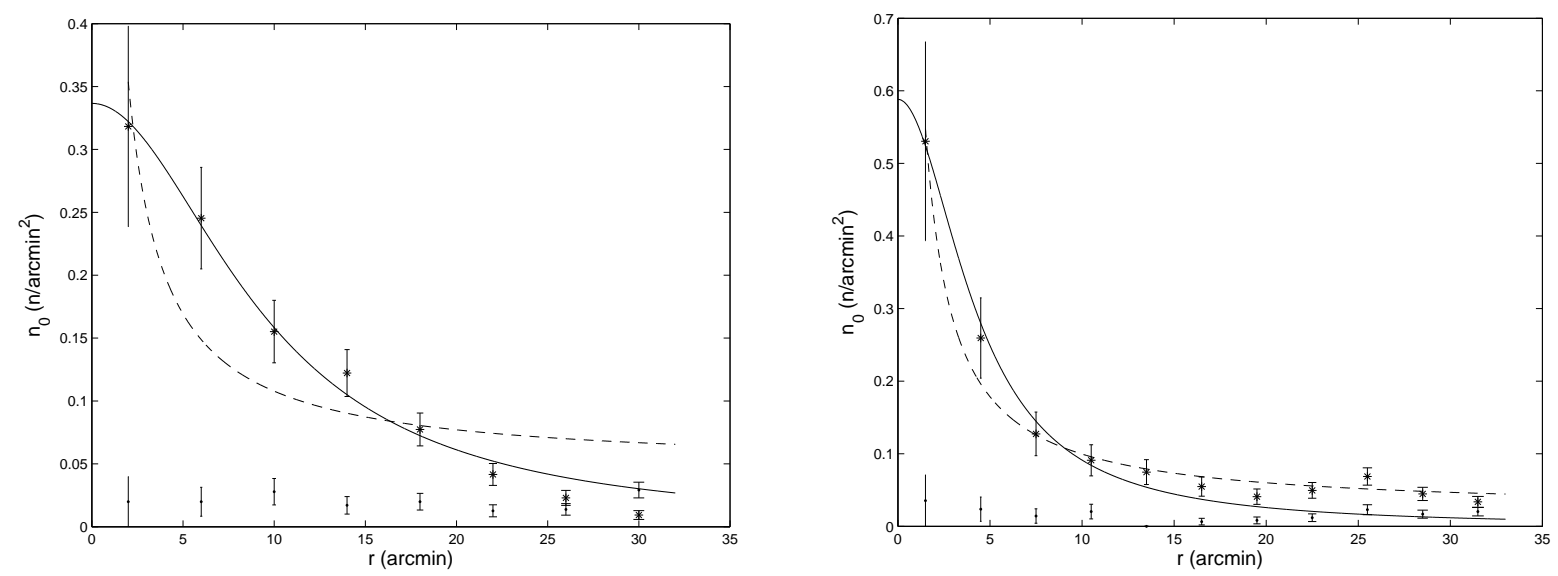

Fig. 3.- Radial number density profiles of the stars in the open cluster regions. Left: NGC 2244; Right: NGC 6530. *: field stars with membership probabilities $p \leq 0.5 ; \cdot$ member stars with $p \geq 0.9$. Solid lines: Gaussian profiles; dashed lines: $R^{-1}$ profiles.
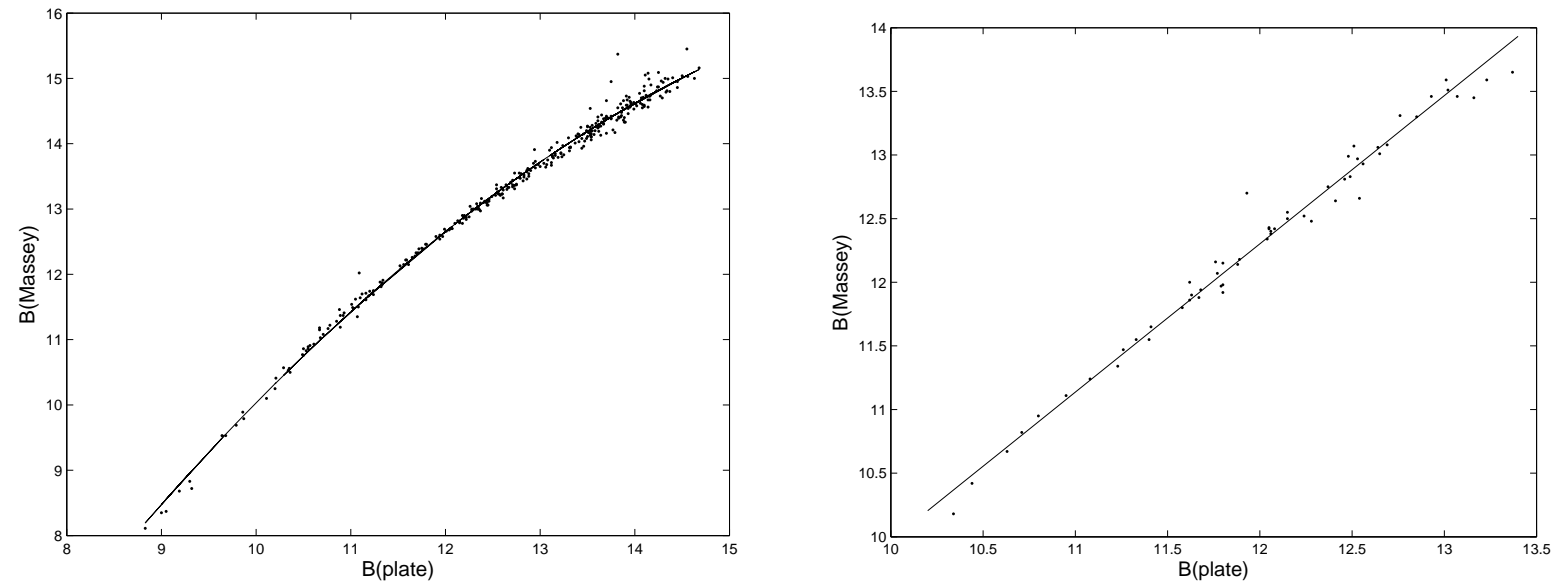

Fig. 4.- Comparison between our instrumental $B_{p}$ photometry and the $B$ magnitudes of the $U B V$ system. Left: 323 stars in common with Massey et al. (1995); Right: 58 stars in common with Prisinzano et al. (2005) 


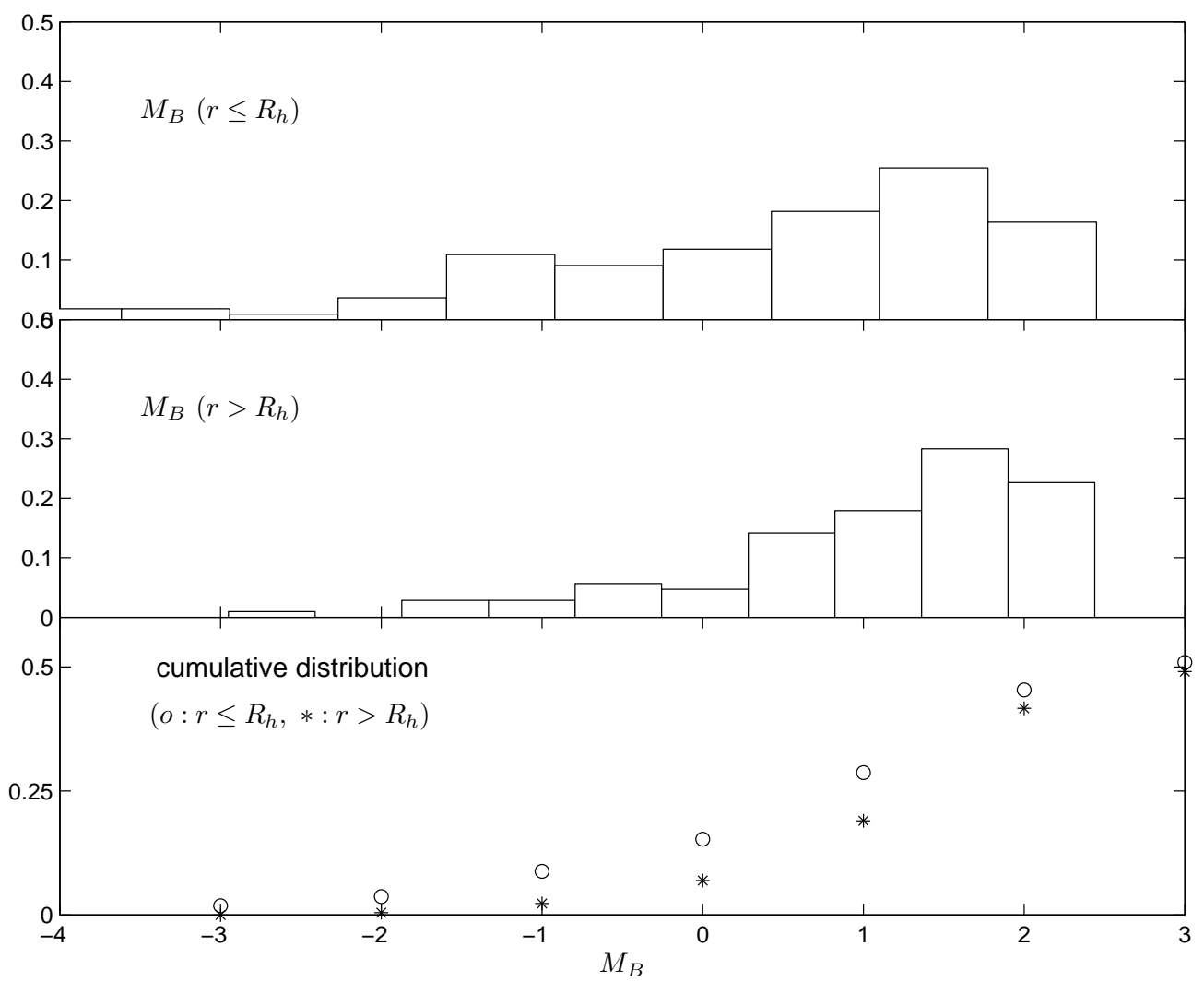

Fig. 5.- Luminosity function of NGC 2244. Top: 110 member stars in the inner area $\left(r \leq 14^{\prime}\right)$; middle: 106 member stars in the outer region $\left(r>14^{\prime}\right)$; bottom: cumulative luminosity functions for both radial regions 


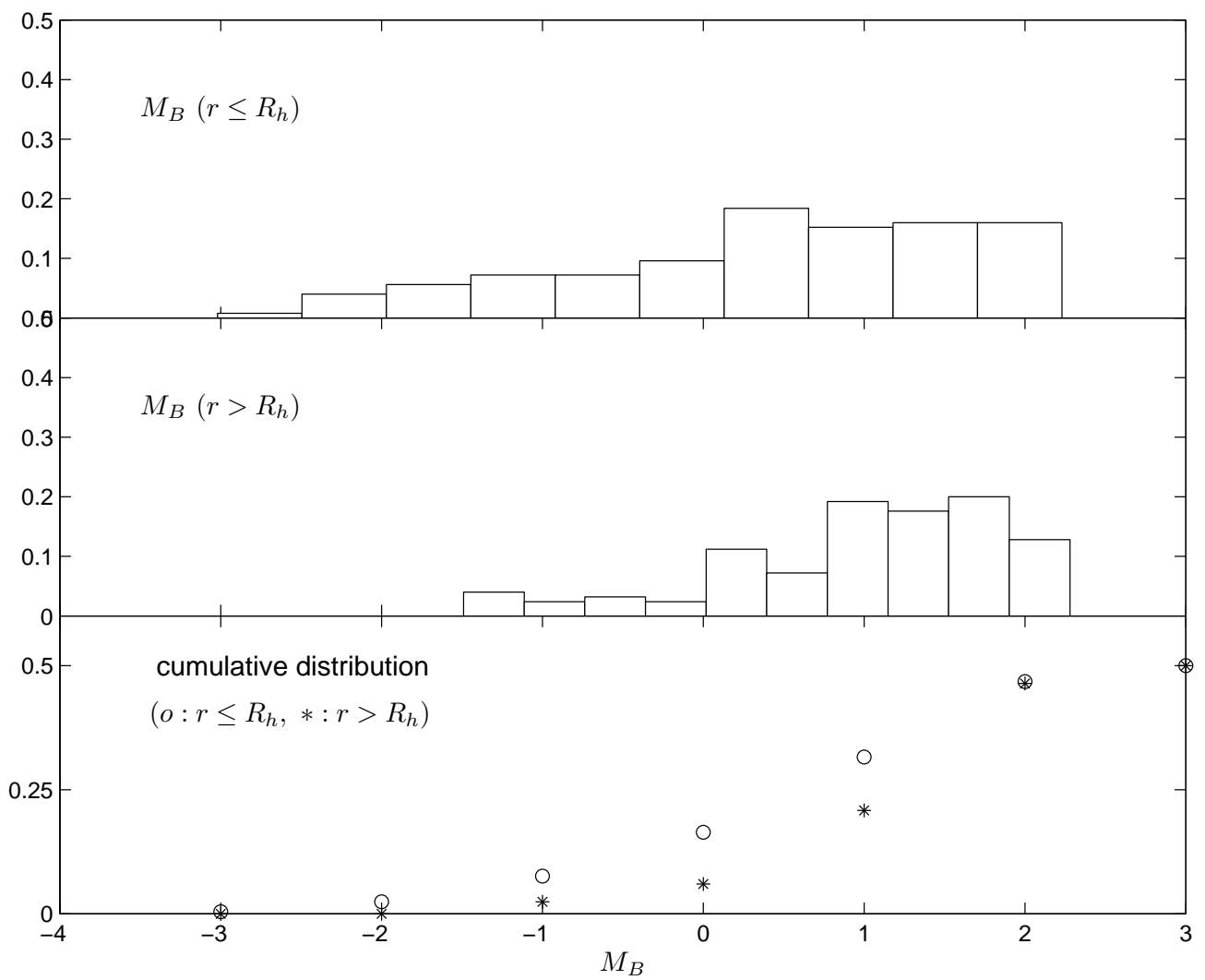

Fig. 6.- Luminosity function of NGC 6530. Top: 125 member stars in the inner area $\left(r \leq 21^{\prime}\right)$; middle: 125 member stars in the outer region $\left(r>21^{\prime}\right)$; bottom: cumulative luminosity functions for both radial regions 


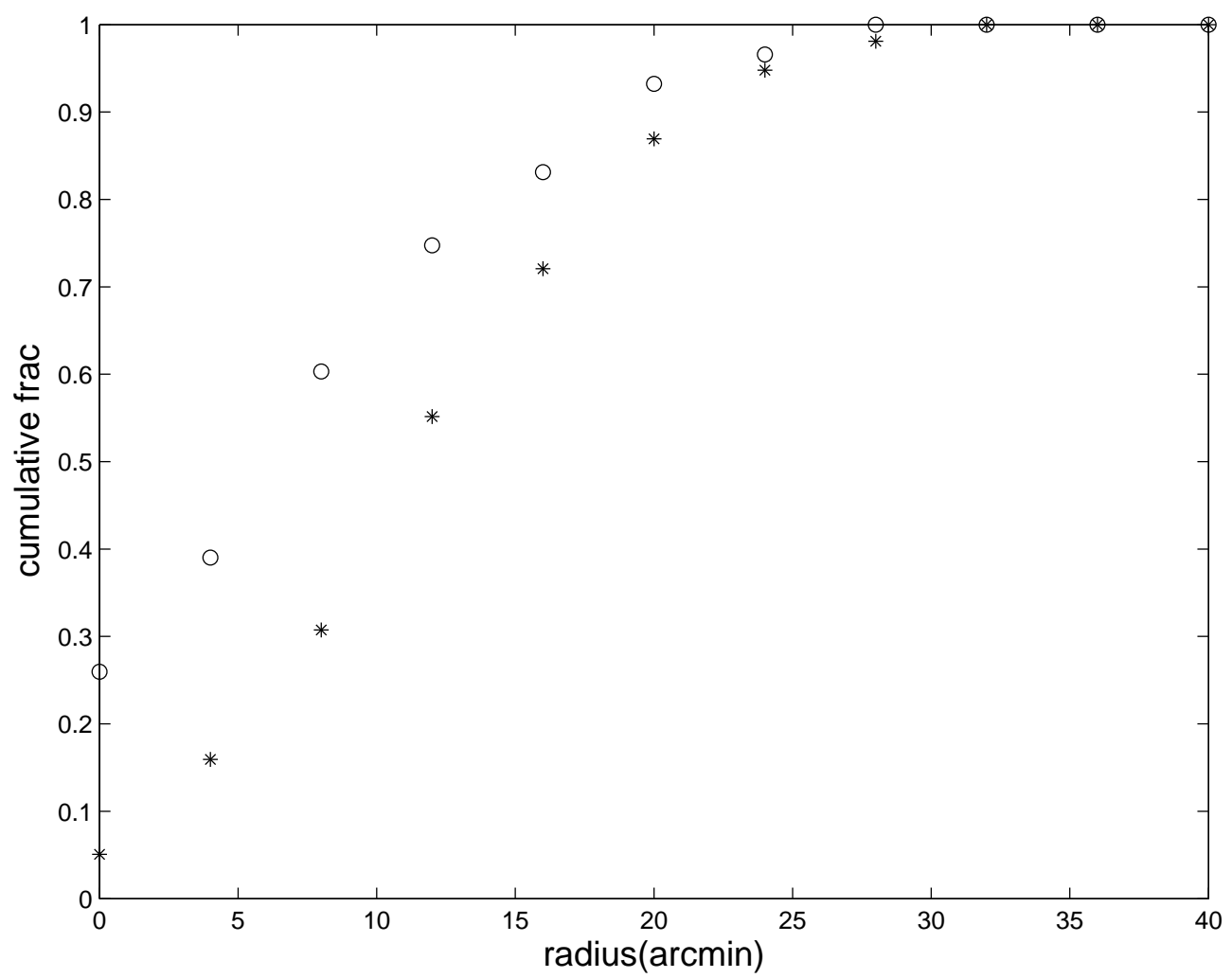

Fig. 7.- Normalized cumulative radial number density profile for NGC 2244 members with $m_{B} \leq 13(\mathrm{o})$ and $m_{B}>13(*)$ 


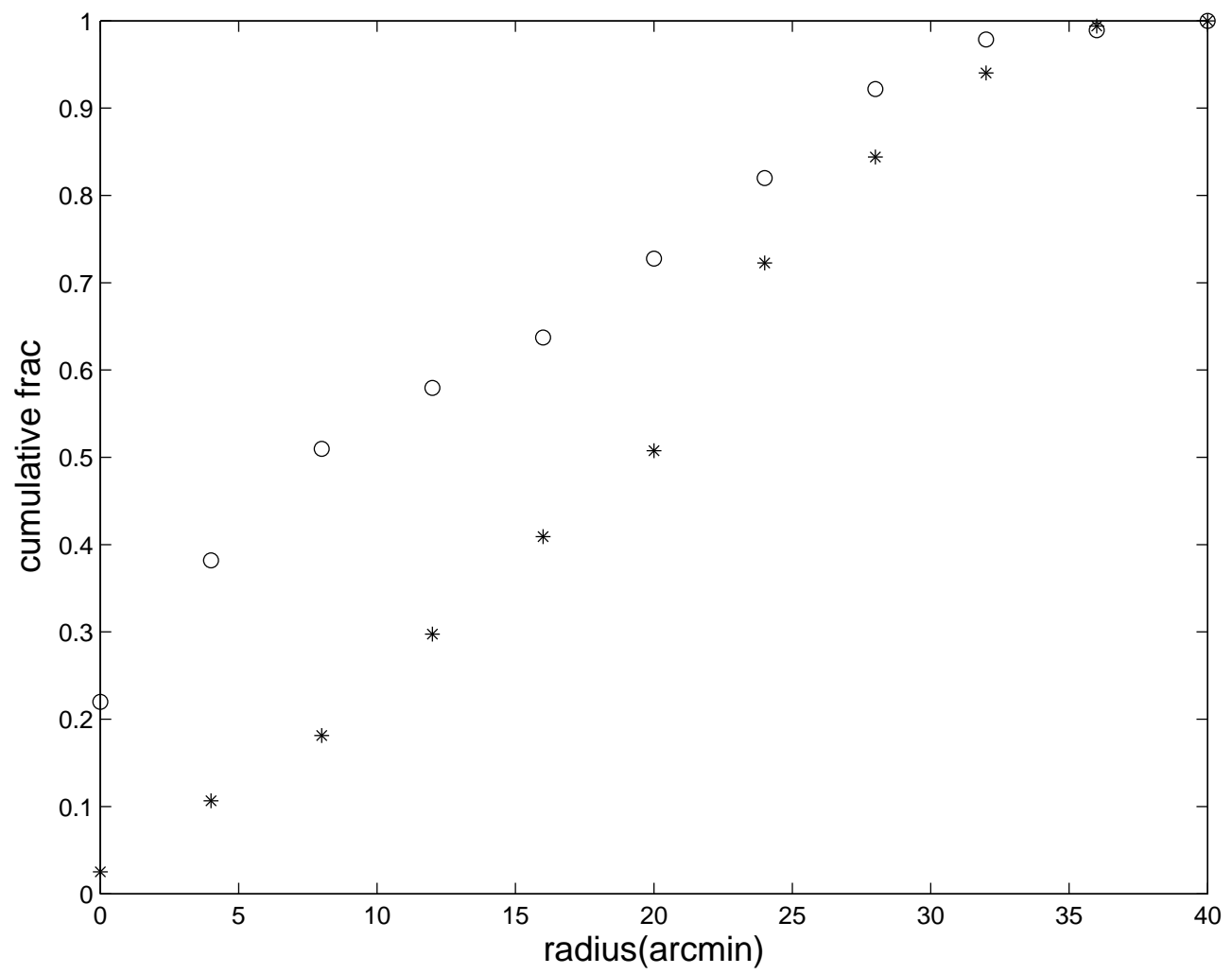

Fig. 8. - Normalized cumulative radial number density profile for NGC 6530 members with $m_{B} \leq 12(\mathrm{o})$ and $m_{B}>12(*)$ 


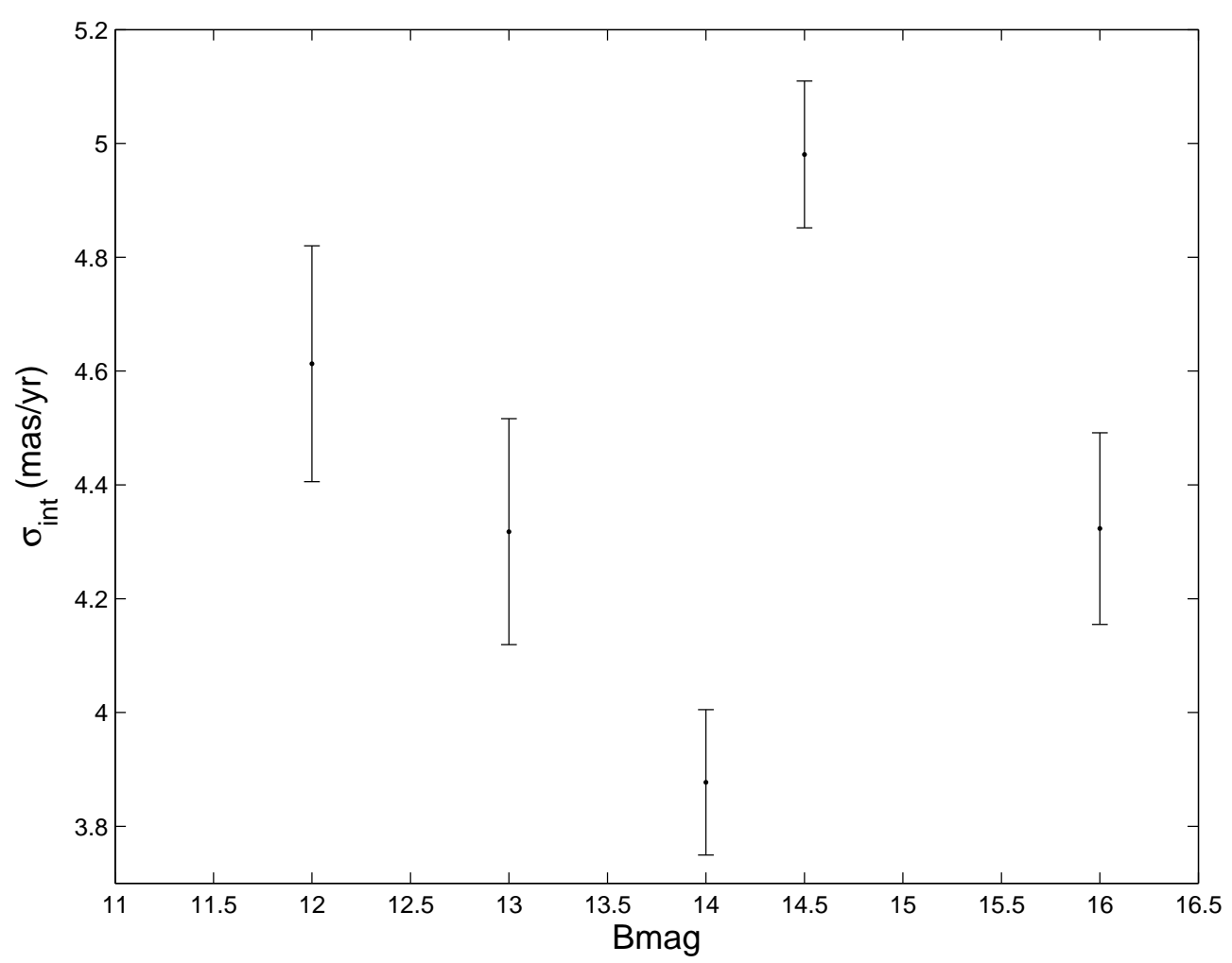

Fig. 9.- Proper motion dispersion of NGC 2244 members as a function of $m_{B}$ magnitude. 


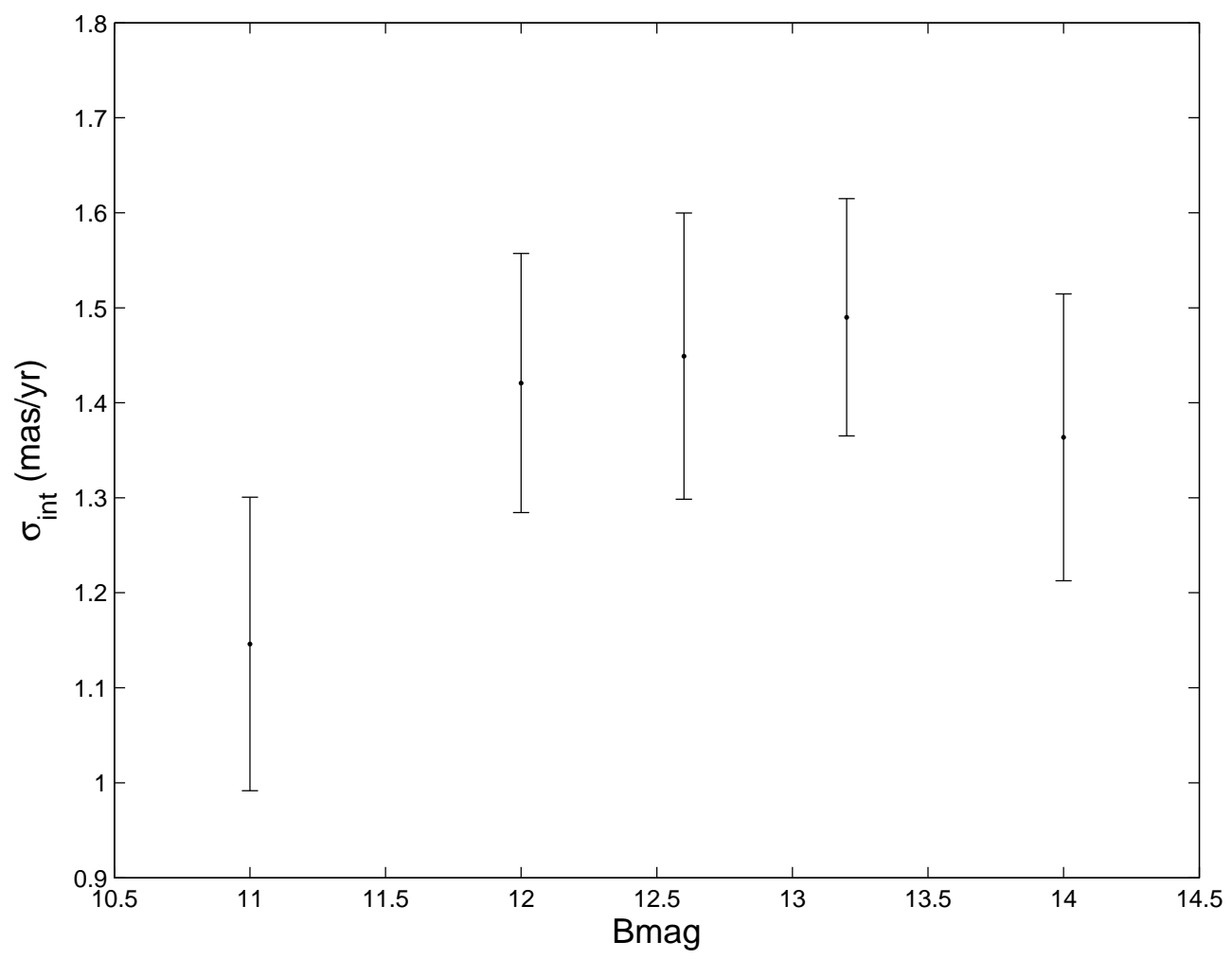

Fig. 10.- Proper motion dispersion of NGC 6530 members as a function of $m_{B}$ magnitude. 
Table 1. Internal levels of uncertainty on the stellar positions and proper motions

\begin{tabular}{|c|c|c|c|c|}
\hline \multirow[b]{2}{*}{ Parameter } & \multicolumn{2}{|c|}{ NGC 2244} & \multicolumn{2}{|c|}{ NGC 6530} \\
\hline & Median error & Maximum error & Median error & Maximum error \\
\hline$\alpha$ & $0^{\mathrm{s}} .003$ & $0^{\mathrm{s}} .017$ & $0^{\mathrm{s}} .003$ & $0^{\mathrm{s}} .014$ \\
\hline$\delta$ & $0^{\prime \prime} .043$ & $0^{\prime \prime} .33$ & $0^{\prime \prime} .06$ & $0^{\prime \prime} .16$ \\
\hline$\mu_{\alpha} \cos \delta$ & $1.39{\mathrm{mas} \mathrm{yr}^{-1}}^{-1}$ & $8.88{\text { mas } \mathrm{yr}^{-1}}^{-1}$ & $0.95 \mathrm{mas} \mathrm{yr}^{-1}$ & $2.74{\mathrm{mas} \mathrm{yr}^{-1}}^{-1}$ \\
\hline$\mu_{\delta}$ & $1.56{\mathrm{mas} \mathrm{yr}^{-1}}^{-1}$ & $9.54 \mathrm{mas} \mathrm{yr}^{-1}$ & $1.21 \mathrm{mas} \mathrm{yr}^{-1}$ & $4.05{\mathrm{mas} \mathrm{yr}^{-1}}^{-1}$ \\
\hline
\end{tabular}


Table 2. Estimates of the distribution parameters

\begin{tabular}{cccc}
\hline \hline & NGC 2244 & & NGC 6530 \\
\cline { 2 - 2 } Parameter & Estimate and uncertainty & & Estimate and uncertainty \\
\hline$\mu_{x \mathrm{c}}\left(\mathrm{mas} \mathrm{yr}^{-1}\right)$ & $-2.51 \pm 0.23$ & & $2.59 \pm 0.16$ \\
$\mu_{y \mathrm{c}}\left(\mathrm{mas} \mathrm{yr}^{-1}\right)$ & $-3.02 \pm 0.25$ & & $-3.48 \pm 0.17$ \\
$\mu_{x \mathrm{f}}\left(\mathrm{mas} \mathrm{yr}^{-1}\right)$ & $-0.41 \pm 0.38$ & & $3.62 \pm 0.34$ \\
$\mu_{y \mathrm{f}}\left(\mathrm{mas} \mathrm{yr}^{-1}\right)$ & $-2.81 \pm 0.28$ & & $-7.69 \pm 0.88$ \\
$\sigma_{0}\left(\operatorname{mas~yr}^{-1}\right)$ & $1.48 \pm 0.14$ & & $2.41 \pm 0.40$ \\
$\sigma_{x 0}\left(\operatorname{mas~yr}^{-1}\right)$ & $2.99 \pm 0.25$ & & $9.19 \pm 0.17$ \\
$\sigma_{y 0}\left(\operatorname{mas~yr}^{-1}\right)$ & $4.81 \pm 0.23$ & & $8.71 \pm 0.36$ \\
$\rho$ & $-0.59 \pm 0.04$ & & $24.45 \pm 0.07$ \\
$\alpha\left({ }^{\prime}\right)$ & $13.24 \pm 0.14$ & & $8.84 \pm 0.21$ \\
$g$ & $19.56 \pm 0.79$ & & \\
\hline
\end{tabular}


Table 3. Proper motions, membership probabilities and $B$ magnitude of 495 stars in the region of NGC 2244

\begin{tabular}{|c|c|c|c|c|c|c|c|c|}
\hline No. & R.A. (J2000.0) & Dec (J2000.0) & $\begin{array}{c}\mu_{\alpha} \cos \delta \\
\text { mas } \mathrm{yr}^{-1}\end{array}$ & 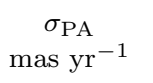 & $\begin{array}{c}\mu_{\delta} \\
\operatorname{mas} \mathrm{yr}^{-1}\end{array}$ & $\begin{array}{c}\sigma_{\mathrm{PD}} \\
\text { mas } \mathrm{yr}^{-1}\end{array}$ & $B$ (mag) & $p$ \\
\hline 1 & 063135.826 & +043051.70 & -6.142 & 0.919 & 4.685 & 0.405 & 8.720 & 0.62 \\
\hline 2 & 063210.470 & +045759.75 & -1.600 & 1.486 & -0.421 & 2.184 & 8.350 & 0.92 \\
\hline 3 & 063136.322 & +045559.53 & 0.336 & 0.464 & -4.710 & 1.008 & 8.830 & 0.89 \\
\hline 4 & 063138.396 & +050136.38 & -1.673 & 0.639 & -0.232 & 1.303 & 8.110 & 0.89 \\
\hline 5 & 063116.839 & +043725.92 & -0.074 & 0.425 & -3.398 & 1.127 & 9.039 & 0.73 \\
\hline
\end{tabular}

Note. - This table is published in its entirety in the electronic edition. A portion is shown here for guidance regarding its form and content. 
Table 4. Proper motions, membership probabilities and $B$ magnitude of 364 stars in the region of NGC 6530

\begin{tabular}{rrrrrrrrr}
\hline \hline No & R.A. (J2000.0) & Dec (J2000.0) & $\begin{array}{c}\mu_{\alpha} \cos \delta \\
\mathrm{mas} \mathrm{yr}^{-1}\end{array}$ & $\begin{array}{c}\sigma_{\mathrm{PA}} \\
\mathrm{mas} \mathrm{yr}^{-1}\end{array}$ & $\begin{array}{c}\mu_{\delta} \\
\mathrm{mas} \mathrm{yr}^{-1}\end{array}$ & $\begin{array}{c}\sigma_{\mathrm{PD}} \\
\mathrm{mas} \mathrm{yr}^{-1}\end{array}$ & $B(\mathrm{mag})$ & $p$ \\
\hline 1 & 180425.848 & -242308.27 & 6.469 & 0.815 & -6.376 & 2.249 & 8.55 & 0.94 \\
2 & 180604.702 & -241143.84 & 5.989 & 0.591 & -8.649 & 1.670 & 8.64 & 0.83 \\
3 & 180415.222 & -241100.07 & 2.958 & 0.877 & -2.713 & 1.067 & 9.12 & 0.99 \\
4 & 180239.798 & -241447.40 & 7.732 & 0.405 & 0.474 & 3.538 & 9.22 & 0.74 \\
5 & 180414.538 & -241436.31 & 8.511 & 0.853 & 9.241 & 1.665 & 9.35 & 0.00 \\
\hline
\end{tabular}

Note. - This table is published in its entirety in the electronic edition. A portion is shown here for guidance regarding its form and content. 
Table 5. Half-number radii of cluster members for different magnitude ranges

\begin{tabular}{cccccc}
\hline \hline & \multicolumn{2}{c}{ NGC 2244} & & \multicolumn{2}{c}{ NGC 6530 } \\
\cline { 2 - 3 } \cline { 5 - 6 }$B$ & $N$ (stars) & Half-number radius & & $N$ (stars) & Half-number radius \\
\hline All member stars & 216 & $13.5^{\prime}$ & & 250 & $20.7^{\prime}$ \\
$M_{B} \leq 0$ mag & 38 & $9.2^{\prime}$ & & 62 & $9.8^{\prime}$ \\
$M_{B}>0$ mag & 178 & $14.3^{\prime}$ & & 188 & $23.2^{\prime}$ \\
\hline
\end{tabular}

